\title{
Strategies to self-manage side-effects of adjuvant endocrine therapy among breast cancer survivors: an umbrella review of empirical evidence and clinical guidelines
}

\author{
Louise H. Hall ${ }^{1}$. Natalie V. King ${ }^{1}$. Christopher D. Graham ${ }^{2}$. Sophie M. C. Green ${ }^{1}$ - Alice Barber ${ }^{3} \cdot$ Richard D. Neal $^{1}$. \\ Robbie Foy ${ }^{1}$. Jane Clark ${ }^{4}$. Kelly E. Lloyd ${ }^{1}$. Samuel G. Smith ${ }^{1}$
}

Received: 6 May 2021 / Accepted: 13 September 2021 / Published online: 18 October 2021

(C) The Author(s) 2021

\begin{abstract}
Purpose Side-effects of adjuvant endocrine therapy (AET) are common in breast cancer survivors, and can affect adherence to treatment. We synthesised the evidence for strategies to self-manage these side-effects.

Methods We searched for systematic reviews and clinical guidelines on self-management strategies for AET side-effects (arthralgia, fatigue, hot flashes, gastrointestinal discomfort, nausea, vulvovaginal symptoms, and sleep disturbance). We searched oncology organisation's websites and eight databases (Inception-November 2020). Screening, data extraction and quality assessment were completed independently in duplicate. PROSPERO: 2019CRD4201914001.

Results We identified 33 systematic reviews and 18 clinical guidelines. $21 \%$ of reviews were high quality, and the average quality score for guidelines was $44 \%$. Evidence for most strategies was absent or weak. There was consensus from a lowquality review and multiple guidelines to recommend moisturisers, gels and lubricants for vulvovaginal symptoms. Evidence was weak for physical activity for self-managing most symptoms, although two high-quality reviews indicated yoga and aerobic exercise could reduce fatigue. Primary research was often biased by weak and underpowered study designs. Eleven reviews did not report information on adverse events.

Conclusions Most self-management strategies for breast cancer survivors experiencing side-effects from AET lack evidence. Primary research is needed using high-quality well-powered designs focusing on implementable strategies.

Implications for Cancer Survivors Patients and clinicians should be aware that although the risk of harm is low for these selfmanagement strategies, the likelihood of benefit is often unclear. Women should consider moisturisers, gels or lubricants for self-managing vulvovaginal symptoms, and yoga or aerobic exercise for alleviating fatigue.
\end{abstract}

Keywords Breast cancer $\cdot$ Adjuvant endocrine therapy $\cdot$ Complementary therapy $\cdot$ Self-management $\cdot$ Systematic review $\cdot$ Side-effects

Samuel G. Smith

s.smith1@leeds.ac.uk

1 Leeds Institute of Health Sciences, University of Leeds, Leeds LS2 9NL, England, UK

2 Department of Psychology, Queen's University Belfast, Belfast BT7 1NN, Northern Ireland, UK

3 School of Medicine, University of Leeds, Leeds LS2 9NL, England, UK

4 Department of Clinical and Health Psychology, St James's University Hospital, Leeds LS9 7TF, England, UK

\section{Introduction}

Breast cancer is the most common cancer in women worldwide, and the leading cause of cancer death [1]. Most tumours are oestrogen-receptor positive $(\mathrm{ER}+)$, which can be treated in the adjuvant setting with endocrine therapies. These include tamoxifen and Aromatase Inhibitors (AIs), such as letrozole, anastrozole, and exemestane. Common side-effects reported by tamoxifen users include hot flashes, fatigue and vulvovaginal symptoms, while arthralgia is commonly reported in women using AIs [2-6]. Most women experiencing these symptoms want more support to manage them [6]. Experiencing these symptoms can reduce quality of life $[2,7-11]$, and is likely to contribute 
to the estimated three-quarters of women who struggle to adhere to and persist with adjuvant endocrine therapy (AET) [12]. Patients and oncologists perceive side-effects to be a major deterrent to AET adherence $[13,14]$.

Women with breast cancer who do not adhere to AET have an increased risk of all-cause and cancer-specific mortality [15-19] and recurrence [16, 20, 21]. It is therefore important to provide evidence-based support to women experiencing side-effects from AET. Women often self-manage these side-effects [6], but use a wide range of strategies which may not be evidence-based. There is a large volume of scientific research and clinical guidelines available on this topic. Therefore, an overall examination of this body of information is needed to identify consistencies and discrepancies in recommendations, and to provide patients and healthcare professionals with clear evidence-based advice for managing these symptoms [22]. We undertook a systematic review of systematic reviews and clinical guidelines (also called an umbrella review) to synthesise evidence for self-management strategies for side effects among women using AET. This approach provides a method for summarising a broad topic of related issues, thereby providing a single source of information for patients, healthcare professionals and research scientists. The inclusion of clinical guidelines within the review will enable us to observe the extent to which evidence is being translated into clinical practice recommendations, to identify self-management strategies recommended by clinical guidelines that are not supported by evidence, and to assess the quality of guidelines informing clinical care.

\section{Methods}

We were interested in literature related to individual-level non-pharmacological or lifestyle treatments or management strategies that could be accessed without seeking a prescription or referral from a healthcare professional. These interventions could be compared against no intervention, usual care or other strategies or treatments, including those administered by a healthcare professional. We were interested in systematic reviews and clinical guidelines reporting outcome measures assessing AET side-effects. We created a list of all side-effects associated with tamoxifen, letrozole, anastrozole, and exemestane and presented them to two primary care physicians ( $\mathrm{RF}$ and $\mathrm{RN}$ ). Consensus was reached that the following could be safely managed without initially seeking support from a healthcare professional: arthralgia (including joint pain and joint disorders), gastrointestinal discomfort, fatigue, hot flashes, nausea, vulvovaginal symptoms, and sleep disturbance.
The review was prospectively pre-registered (PROSPERO 2019 CRD4201914001). We adhered to the PRISMA 2020 checklist throughout [23].

\section{Search strategy}

In March 2019, we searched for systematic reviews examining the self-management strategies of common side effects experienced by breast cancer patients being treated with AETs. We updated the searches in November 2020. The following databases were searched from inception to present; CINAHL, Cochrane, Embase, Medline, and Web of Science (Appendix). The database searches were developed for three search concepts: breast cancer; tamoxifen or Aromatase Inhibitors and generic and specific side effects. Database subject headings and free text words were identified by the Information Specialist (NK) and project team members (SS, LH). The search was peer-reviewed by another Information Specialist using the PRESS checklist [24]. To identify relevant clinical guidelines, we searched for AETs and the named side-effects in the following websites in July 2019 and November 2020: American Society for Clinical Oncology (ASCO), European Society of Medical Oncology (ESMO), NIH National Cancer Institute (NCI), National Comprehensive Cancer Network (NCCN), Scottish Intercollegiate Guidelines Network (SIGN), and National Institute for Health and Care Excellence (NICE) Evidence. We also searched the guidelines databases; Turning Research Into Practice (TRIP), International Guidelines Library (GIN), and National Health and Medical Research Council (NHMRC) Australian Clinical Practice Guidelines. The results were stored and deduplicated using Endnote X9 software. Further relevant studies were sought by backward citation searching of the included studies.

\section{Study selection}

Screening was conducted in two stages using Covidence software. Two authors (SS, LH, or ER) independently screened the titles and abstracts for eligibility and conducted the full text screen. Disagreements were resolved through discussion with a third author (CG). We included systematic reviews and meta-analyses and guidelines from societies, government agencies and charities with the following criteria; women with breast cancer using AET (e.g. tamoxifen, aromatase inhibitors, anastrozole, letrozole or exemestane). The interventions were individual-level, non-pharmacological or lifestyle treatments or management strategies available without a prescription or referral from healthcare professionals. They could be compared against usual care, other self-management strategies, or treatments administered by a healthcare professional. We excluded narrative reviews and studies reporting primary data, reviews focussed mainly on 
cancers other than breast cancer, symptoms not associated with AET, switching treatments, the neoadjuvant setting or side-effects that require clinical management.

\section{Data collection}

Data extraction was conducted in Microsoft Excel in duplicate (SS, LH), with a third author (KL) arbitrating any disagreements. The data extraction form was piloted before full data extraction took place. Key study variables extracted for the systematic reviews are shown in Table 1. We also extracted information on study designs eligible, approach to synthesis, self-management strategies reported, sample size and effect sizes in meta-analysis, key findings and limitations. Key study variables extracted for the clinical guidelines are shown in Table 2.

\section{Evidence synthesis}

Studies and guidelines were summarised in tables, and were classified into 3 categories: ' $\mathrm{X}$ ' $=$ Overall, no evidence or low-quality evidence in support of the intervention or high-quality evidence against the intervention; '?'= mixed evidence with some evidence in favour of the intervention and some negative or null evidence against the intervention or no evidence provided for recommendation; ' $\checkmark$ ' $=$ Overall, more evidence or higher quality evidence in favour of the intervention than against it. We also undertook a narrative synthesis of the data. We first synthesized the data from the systematic reviews and guidelines independently, and then combined them to identify consistencies and discrepancies in terms of the strategies evaluated, and the conclusions drawn. There was high heterogeneity across the reviews and guidelines with regard to quality, strategies and side-effects included, and therefore no further quantitative synthesis was performed. We grouped reviews and guidelines thematically by side-effects, and self-management strategies reported. Higher quality reviews and guidelines were prioritised in the narrative synthesis when multiple data sources were available. However, lower quality reviews and guidelines were included to increase the sensitivity for identifying potential self-management strategies, and to enable us to provide recommendations for how progress can be made in this field.

The quality of systematic reviews was independently assessed using the AMSTAR-2 checklist by two authors (SS and $\mathrm{LH}$ ), with disagreements resolved by a third author (KL). Seven domains assessed to produce a single quality score of critically low, low, moderate and high [25]. These include protocol registration, adequacy of the search, exclusion criteria justification, risk of bias from individual studies, appropriateness of meta-analytical methods, consideration of risk of bias when interpreting results, and assessment and impact of publication bias. Three authors (KL, SS and AB) independently used the AGREE- 2 checklist to assess the quality of the clinical guidelines across six domains [26]. These include scope and purpose, stakeholder involvement, rigour of development, clarity of presentation, applicability, editorial independence. An average of their scores was computed. Guidelines with scores $>50 \%$ were considered 'higher quality'.

\section{Changes from pre-registration}

We did not extract data related to medication adherence, or broader outcomes related to side-effects such as quality of life or physical function. We also assessed the quality of the clinical guidelines using the AGREE-2 checklist. Some clinical guidelines also had separate document intended for patients rather than healthcare professionals. Where these included broadly identical content, we only included the healthcare professional versions.

\section{Results}

\section{Description}

The final database searches for systematic reviews retrieved 906 records. After duplicates were removed 580 titles and abstracts were screened and 70 full texts were reviewed. Thirty-three systematic reviews were eligible, including 10 identified via backward citation searching (Supplementary Figure S1). We identified 1426 clinical guidelines, and after deduplication 953 titles were screened, and 122 full guidelines were reviewed. Eighteen clinical guidelines were eligible, including one guideline identified via backwards citation searching (Supplementary Figure S2).

The quality of the systematic reviews was mixed. Four systematic reviews had critically low quality [27-30], nine were low quality [31-39], 13 moderate quality [40-52], and seven high quality [53-59] (Table 1). There were consistent weaknesses within the reviews. The quality assessment ratings for each systematic review are available in Appendix Table S1 and S2. No reviews reported the source of study funding, and pre-registrations and explanations for deviating from protocols was uncommon (six adherent [50, 54, 56-59], one partially adherent [40]). Six reviews failed to disclose author conflicts of interest or how they managed existing conflicts [29, 30, 32, 33, 46, 48]. The mean overall quality score of the clinical guidelines was $44 \%$, ranging from 24 [60] to 71 [61] (Table 3). Guidelines scored particularly strongly on the 'scope and purpose' $($ mean $=72 \%)$ and 'clarity of presentation' (mean $=71 \%)$ domains, but 'applicability' $($ mean $=11 \%)$ and 'editorial independence' (24\%) was lower. 


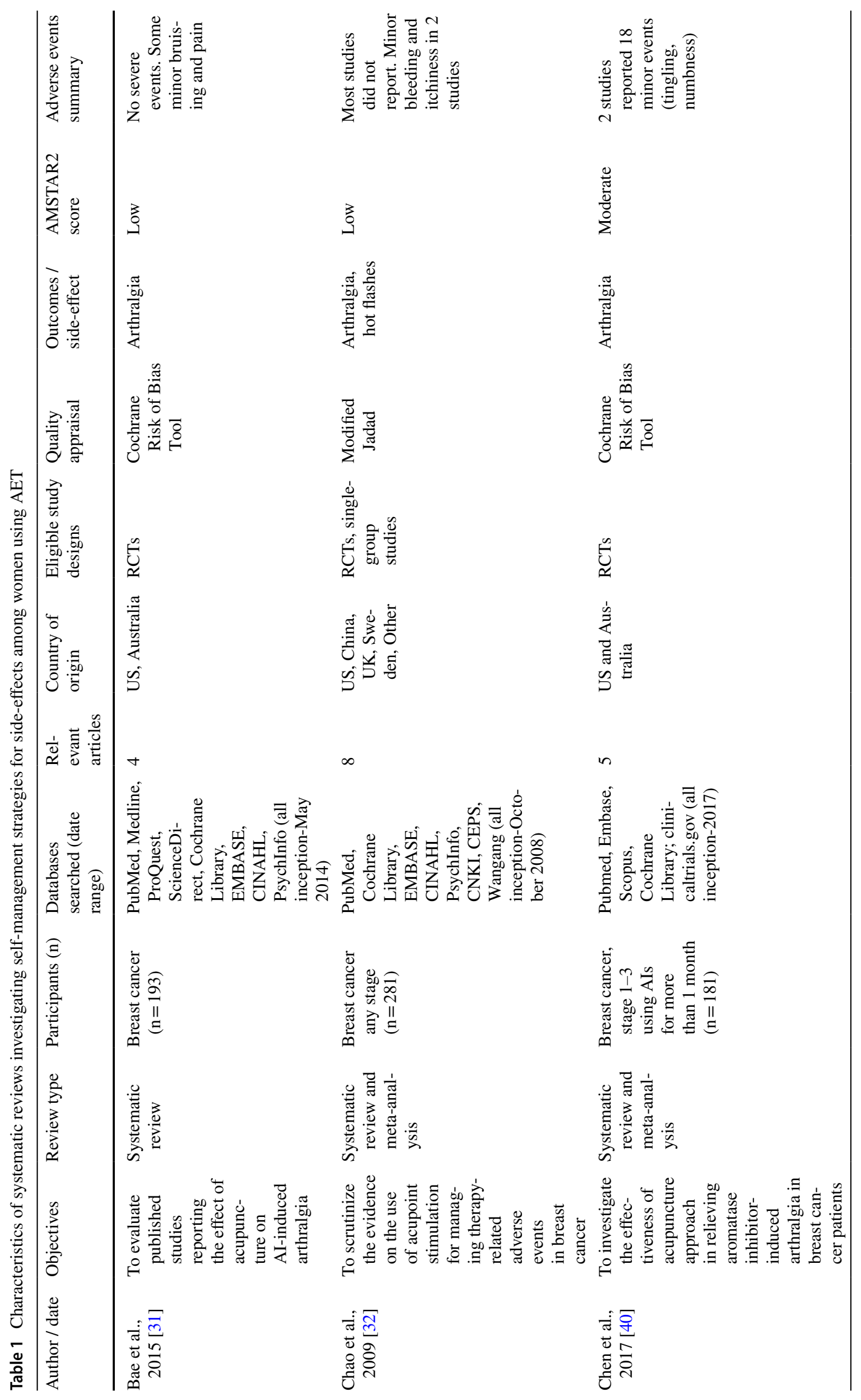




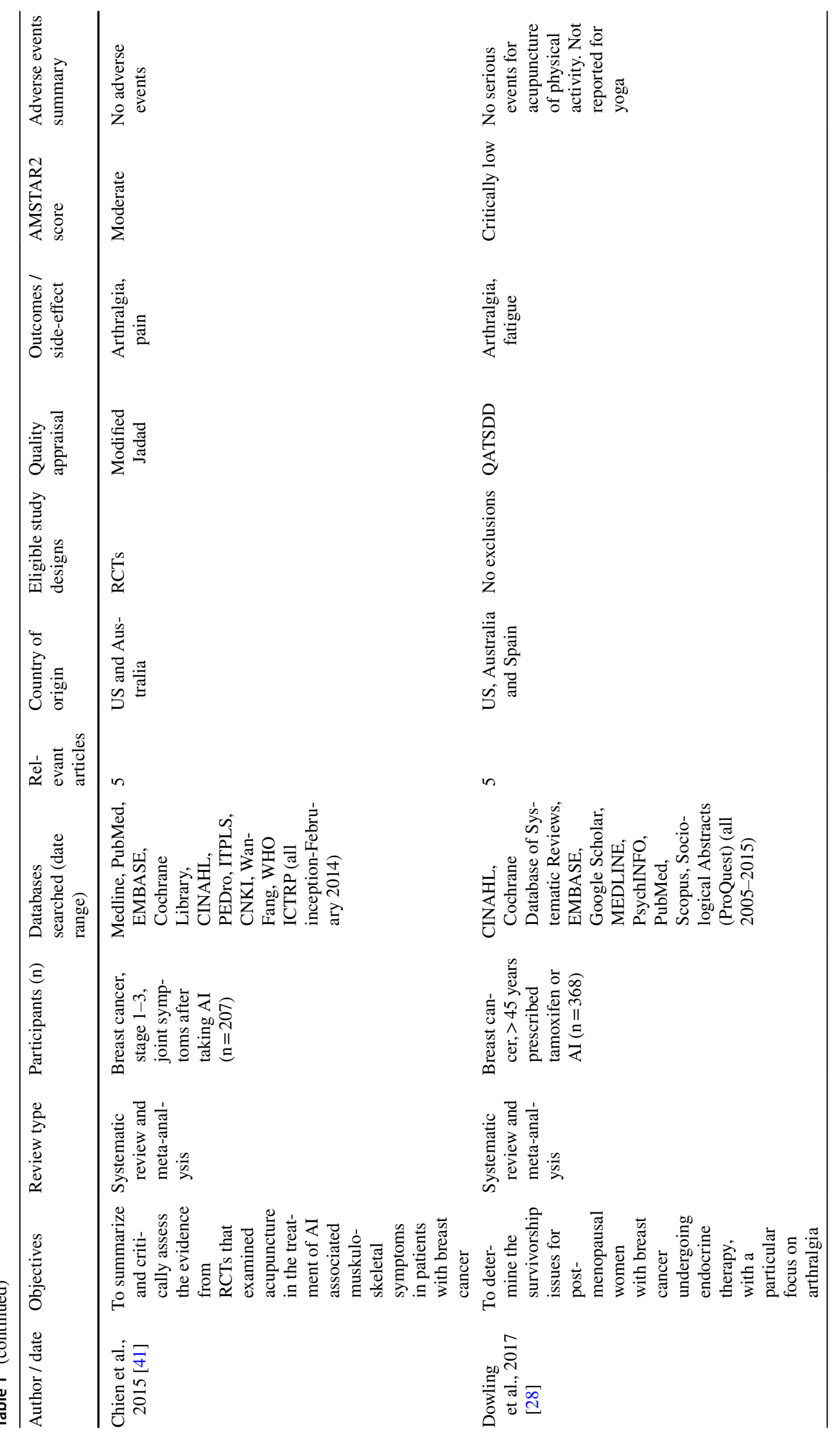




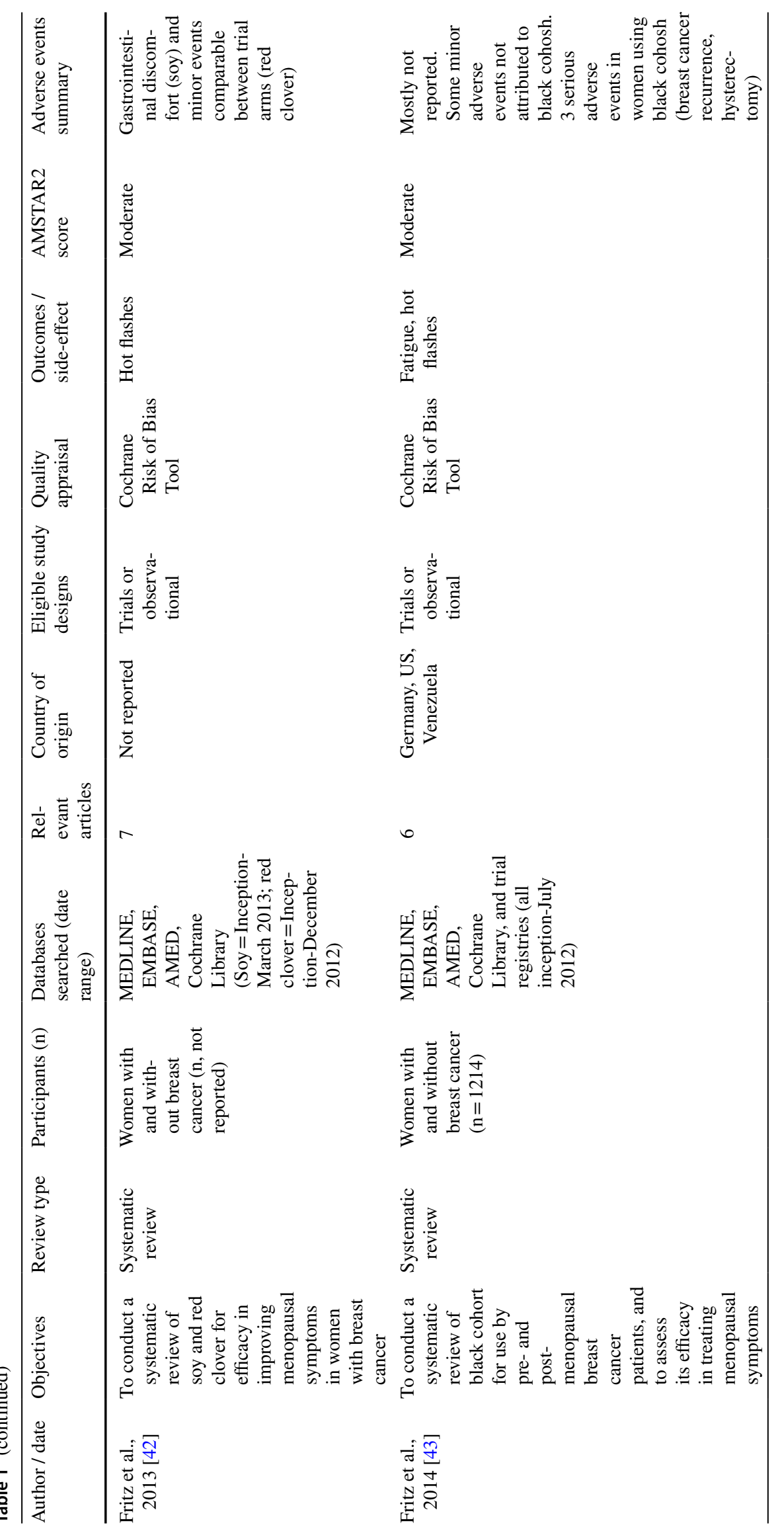




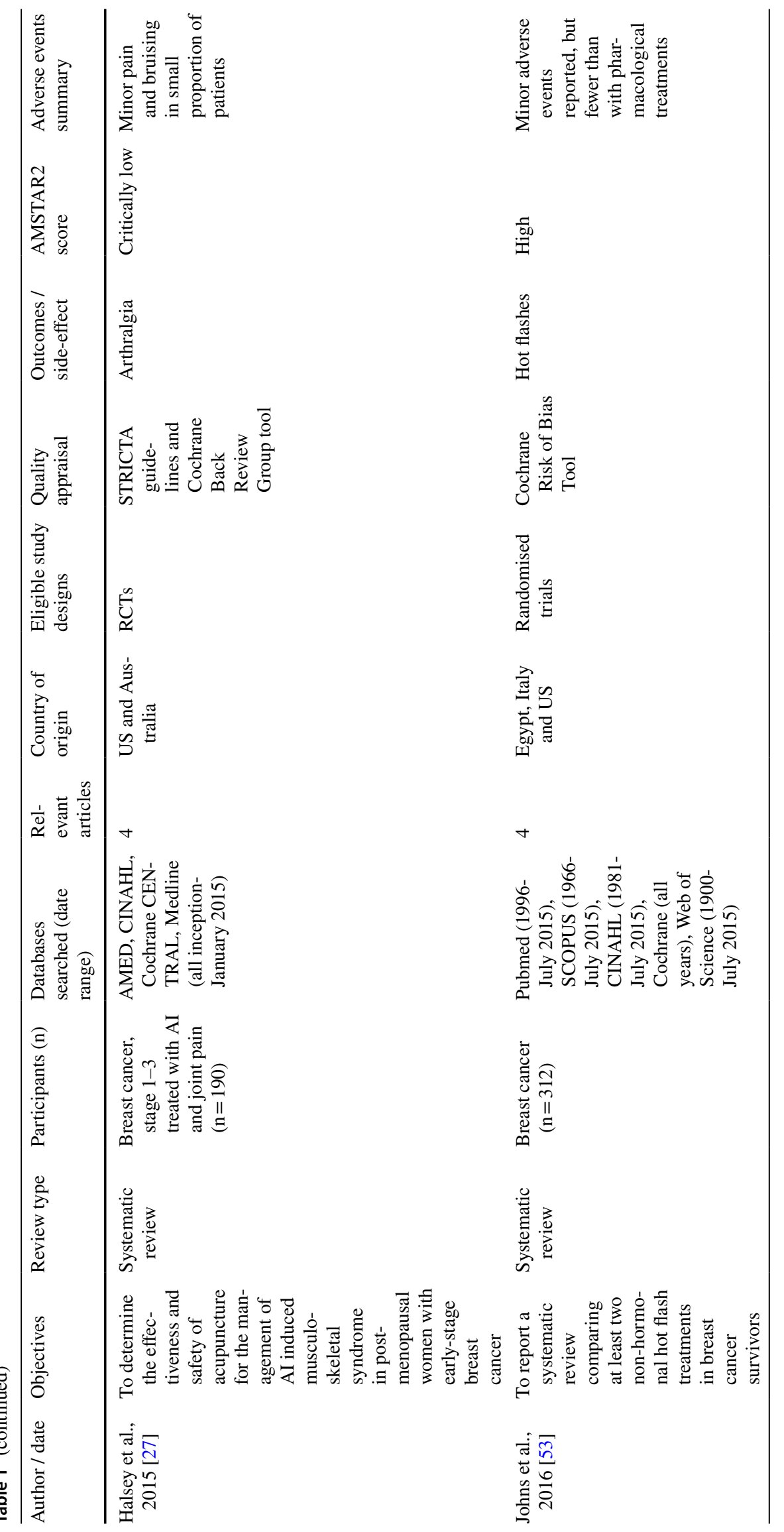




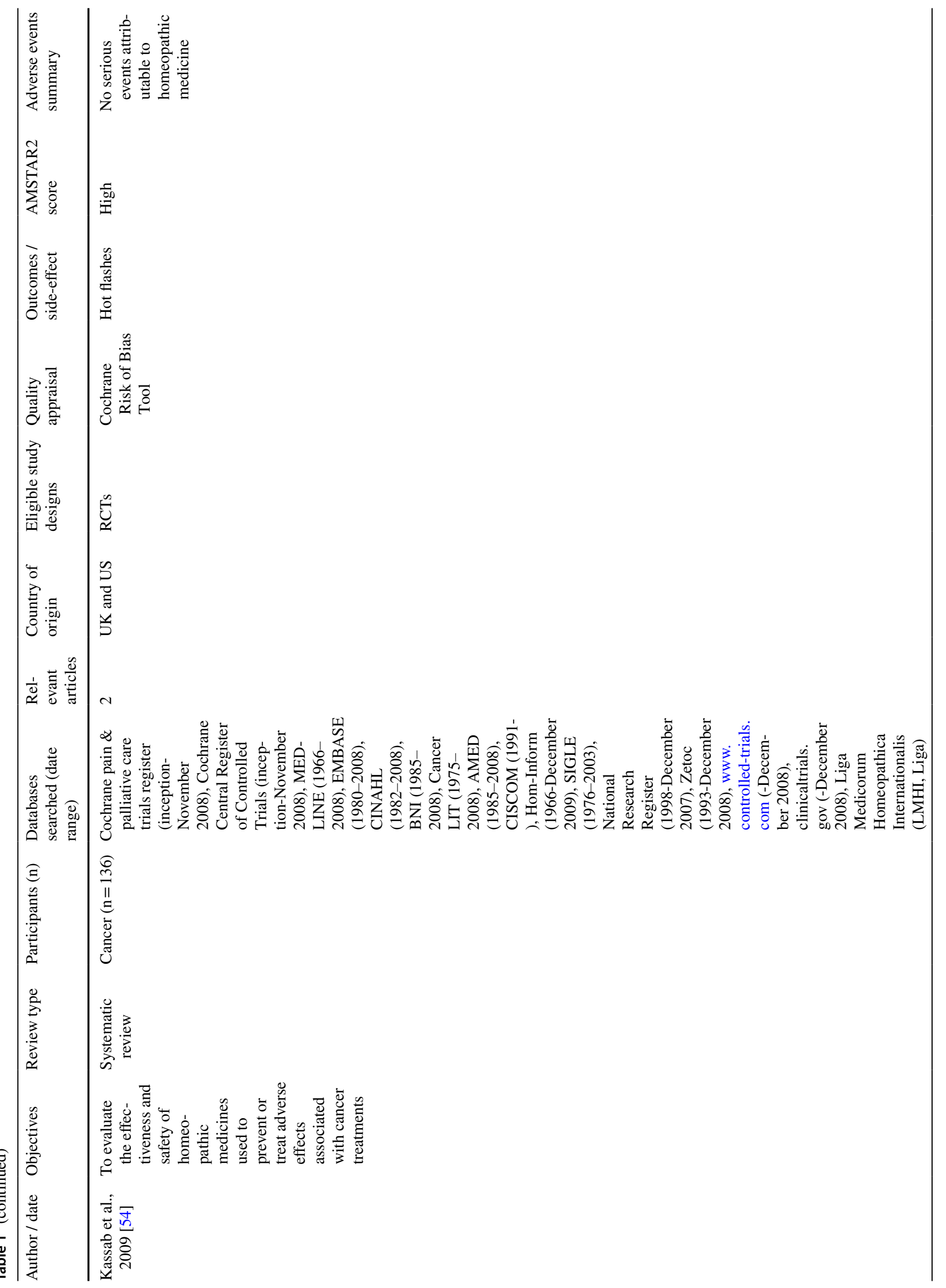




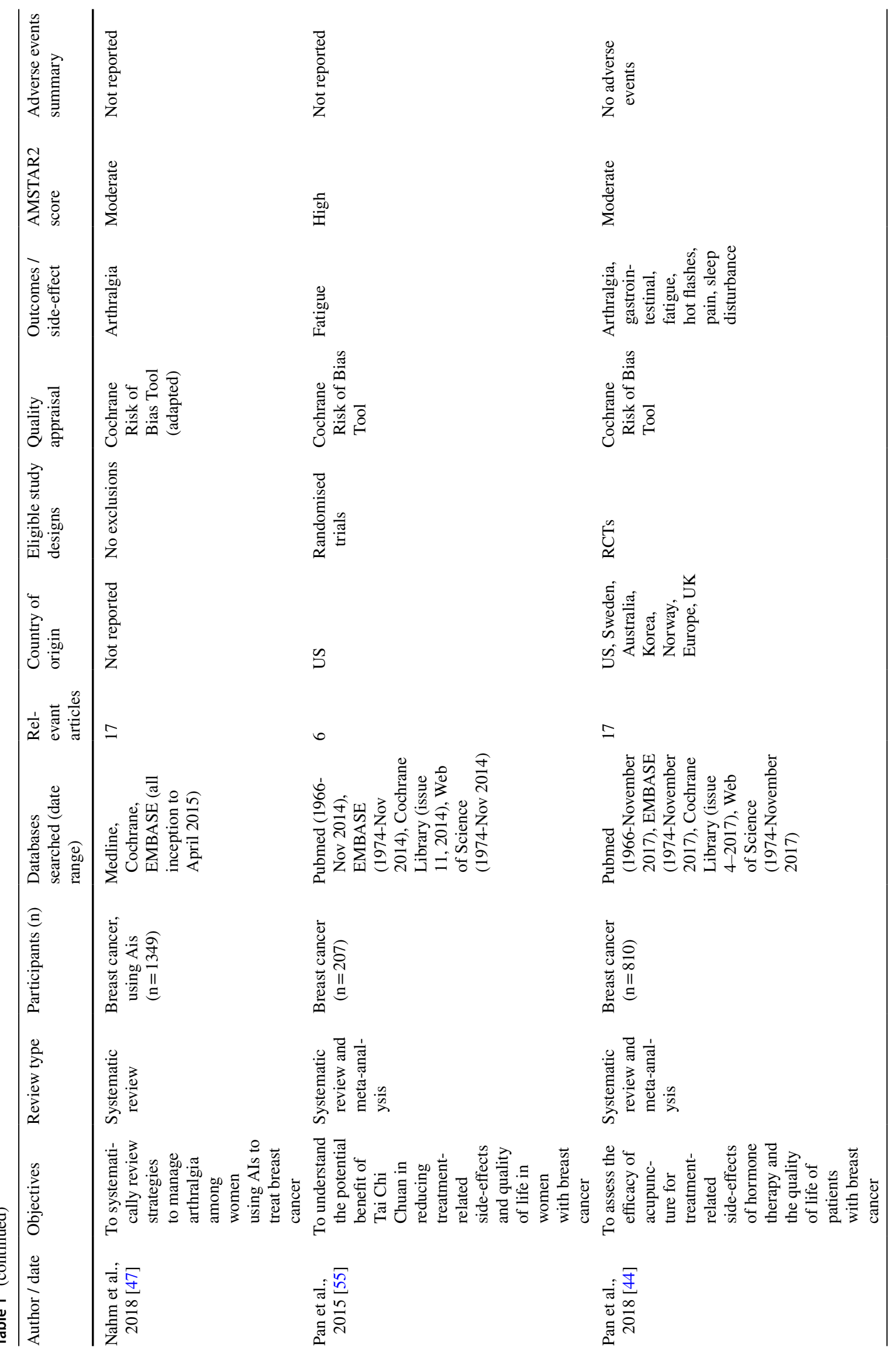




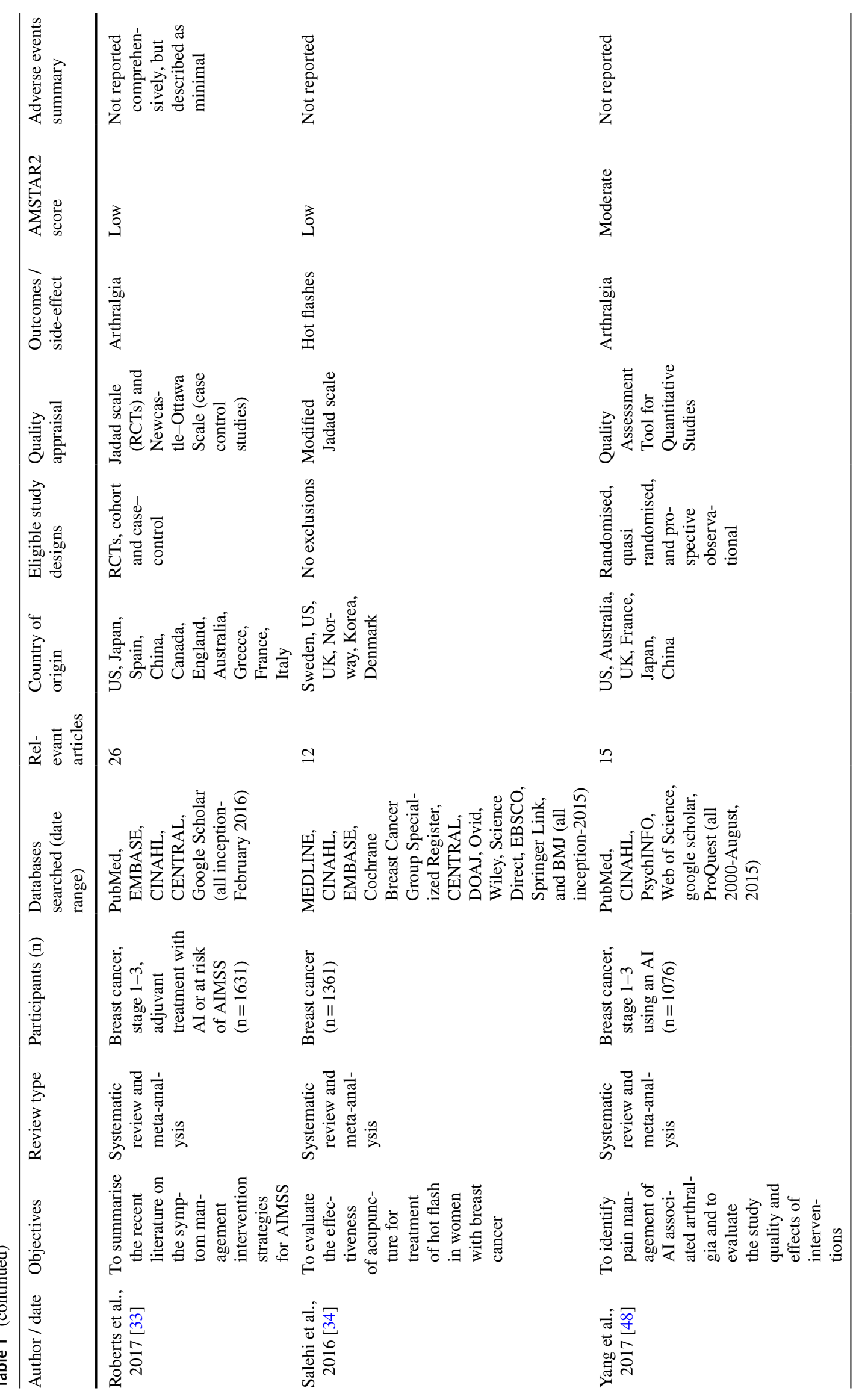




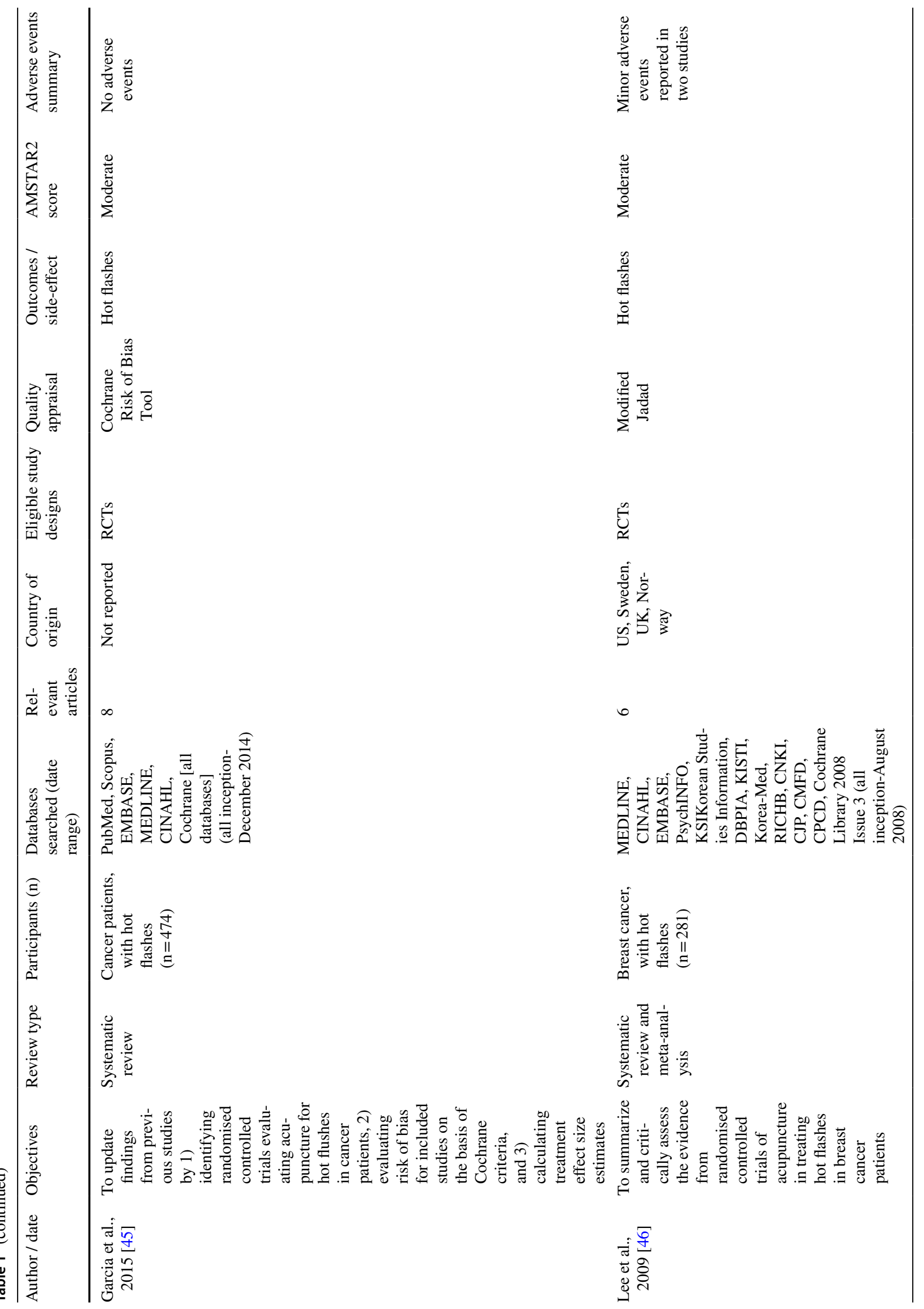




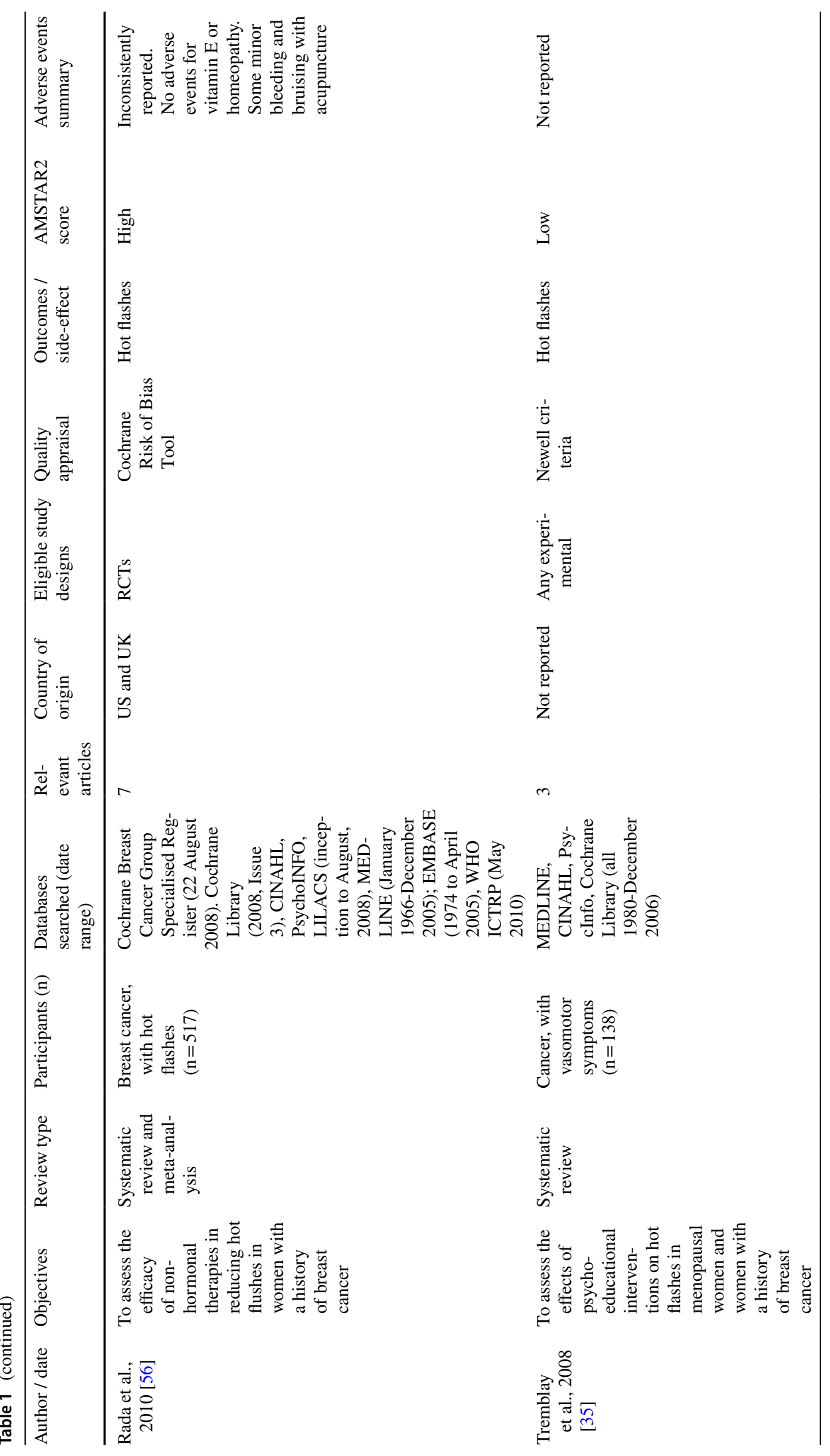




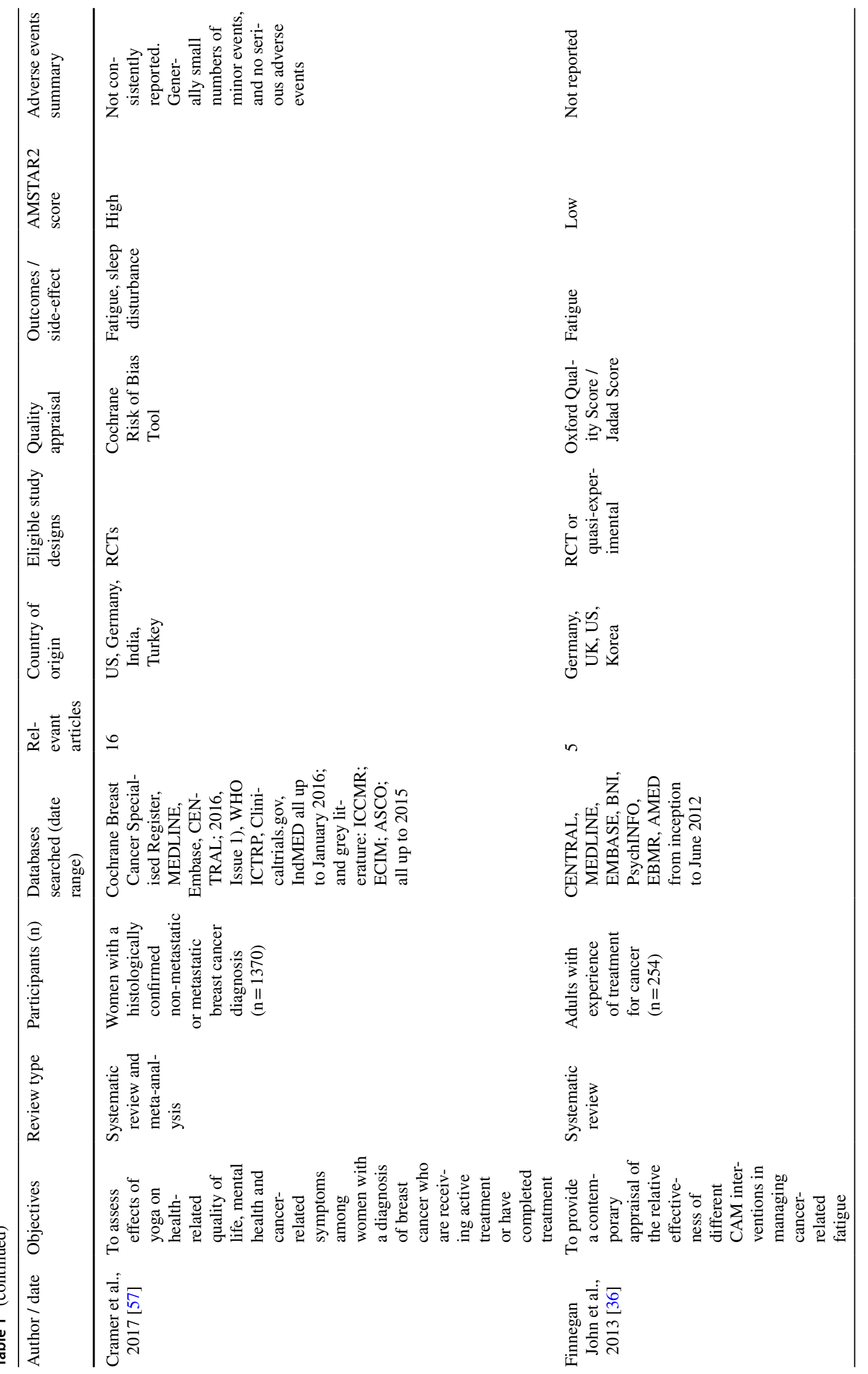




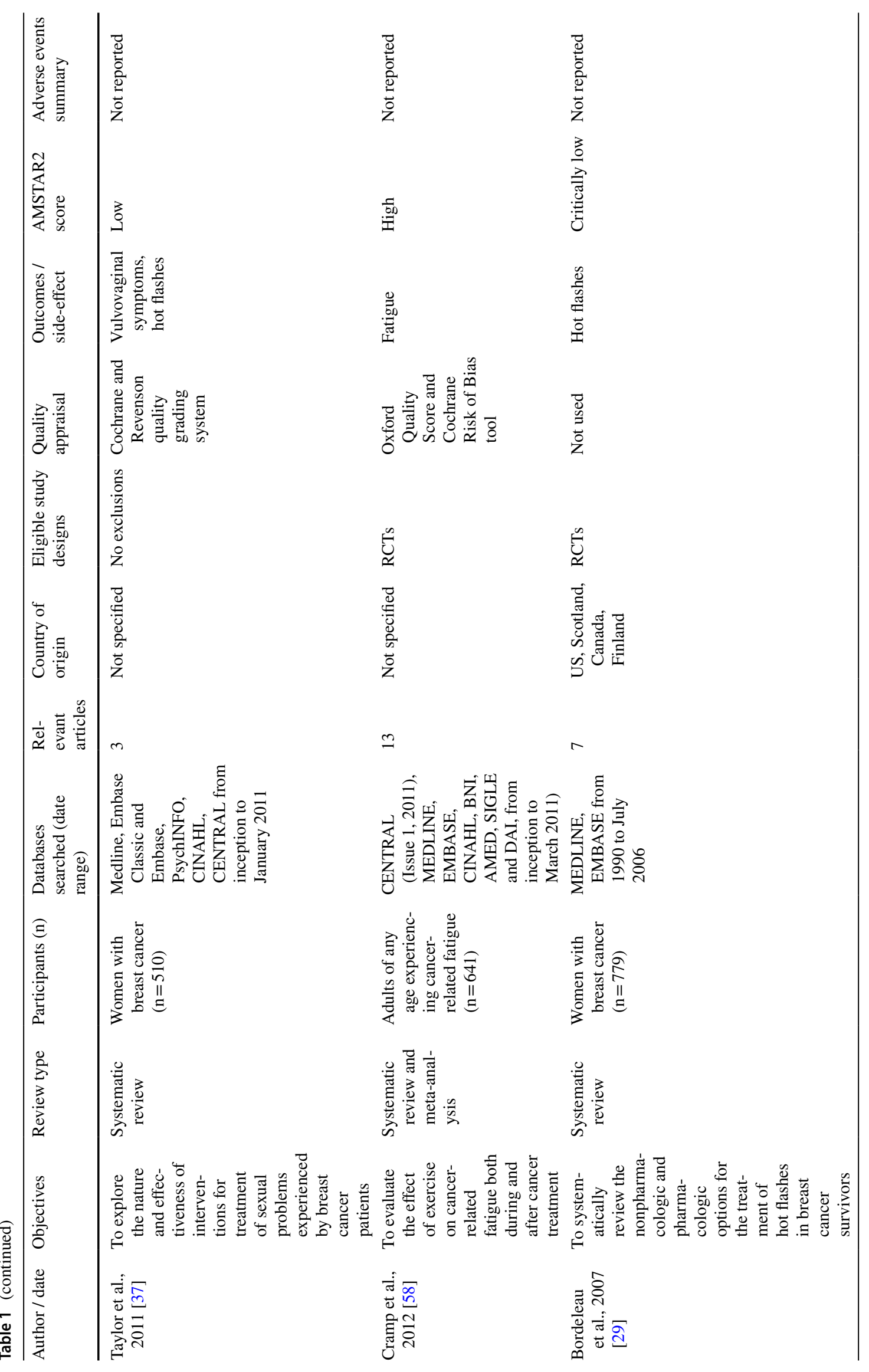




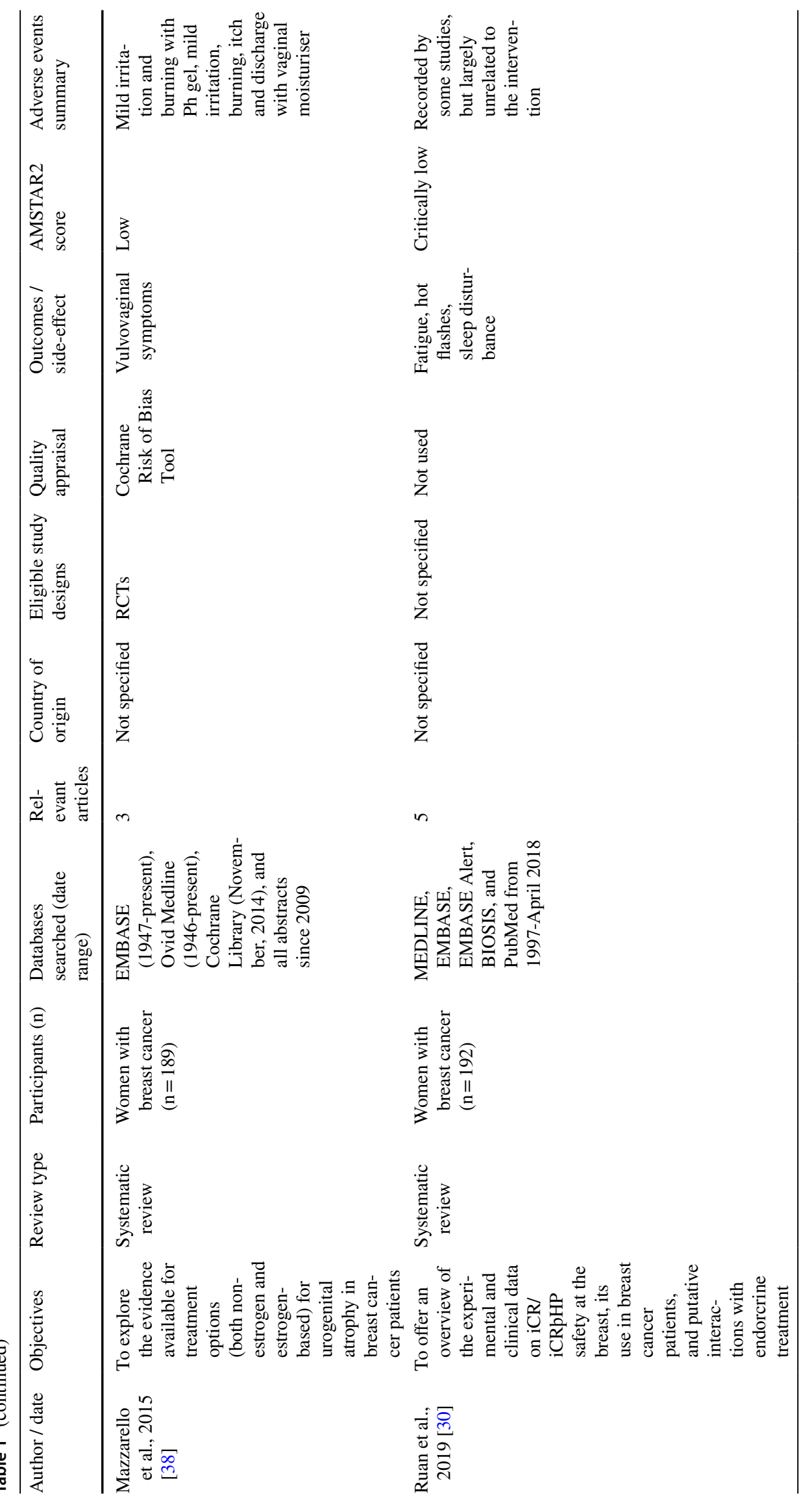




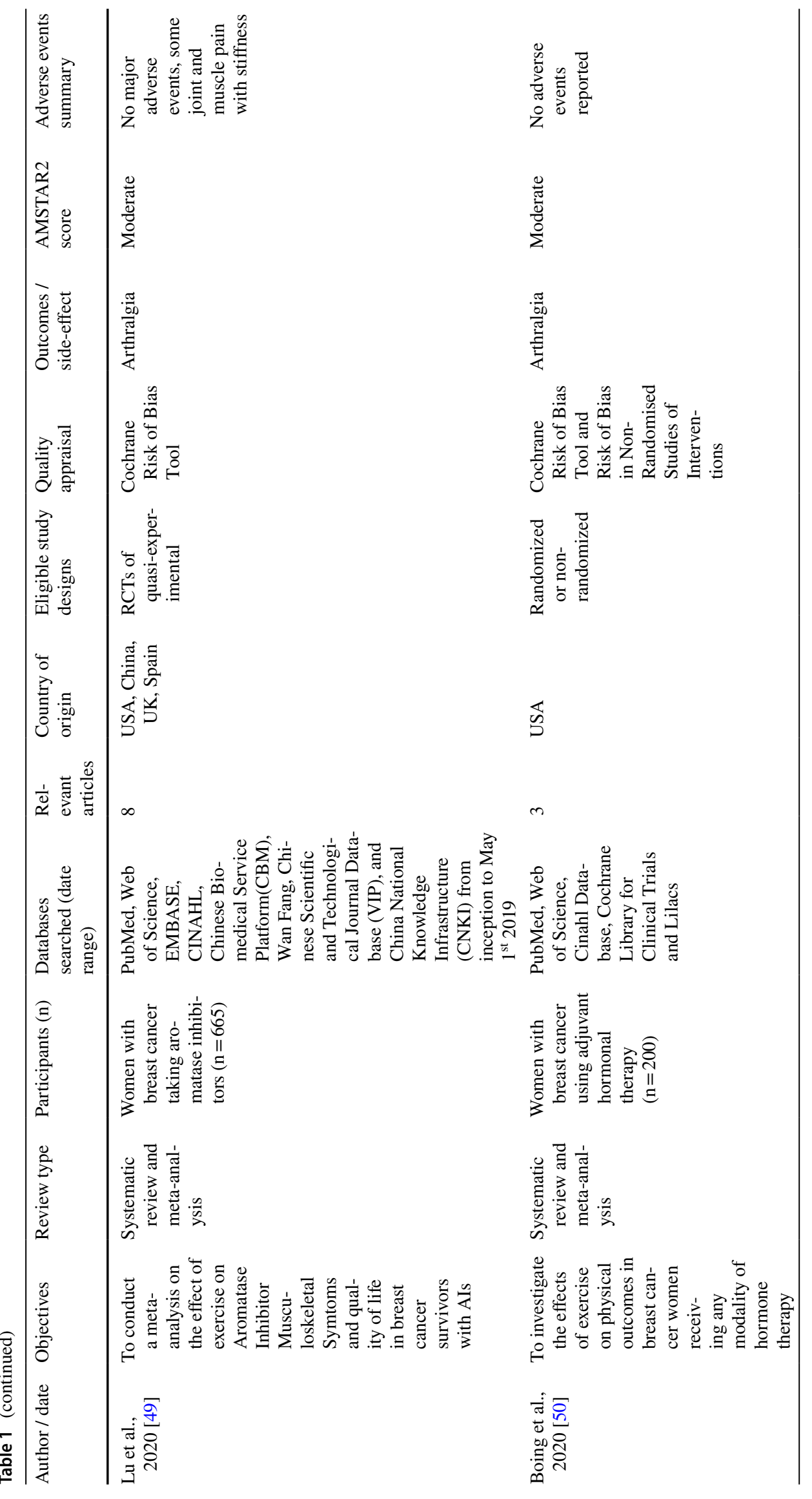




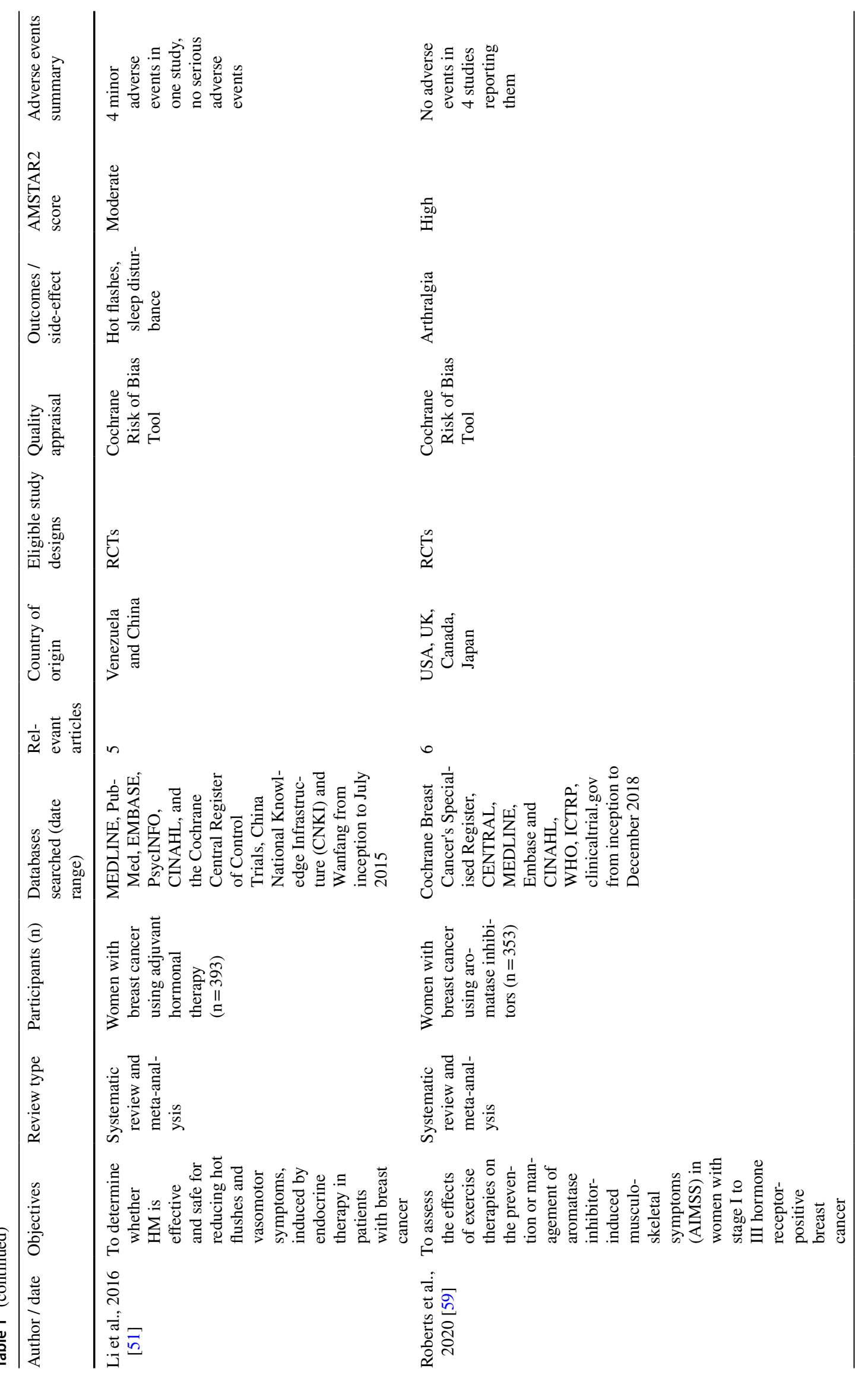




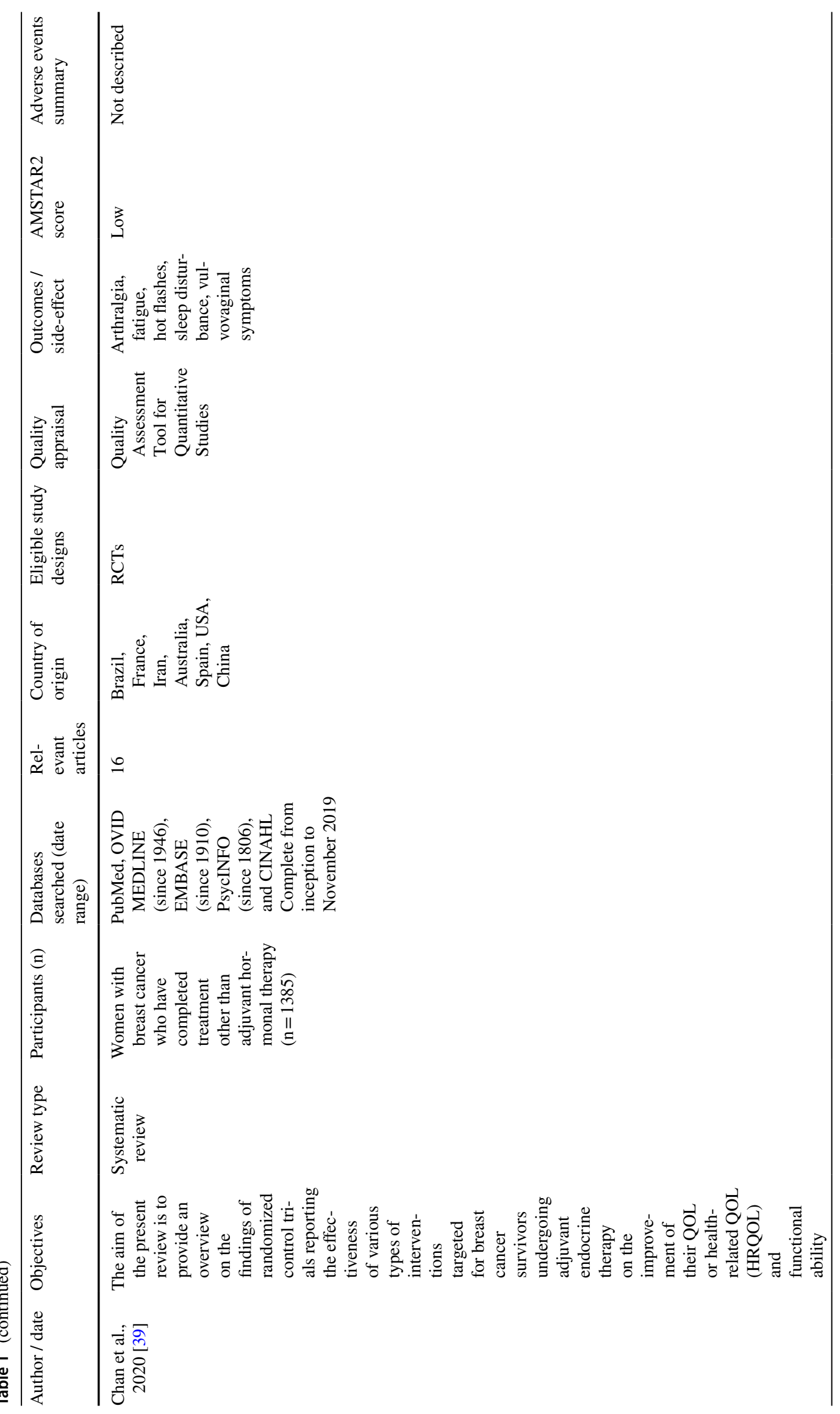




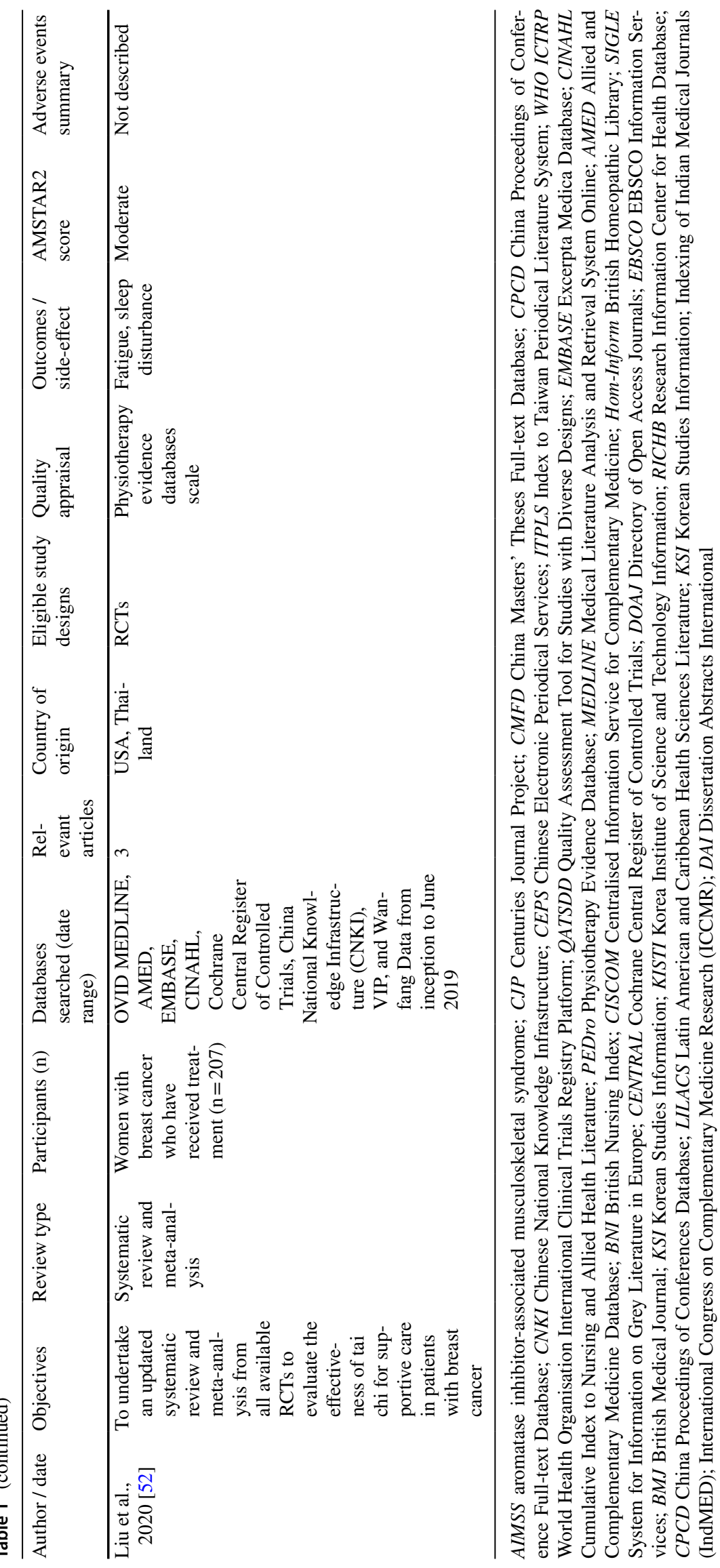




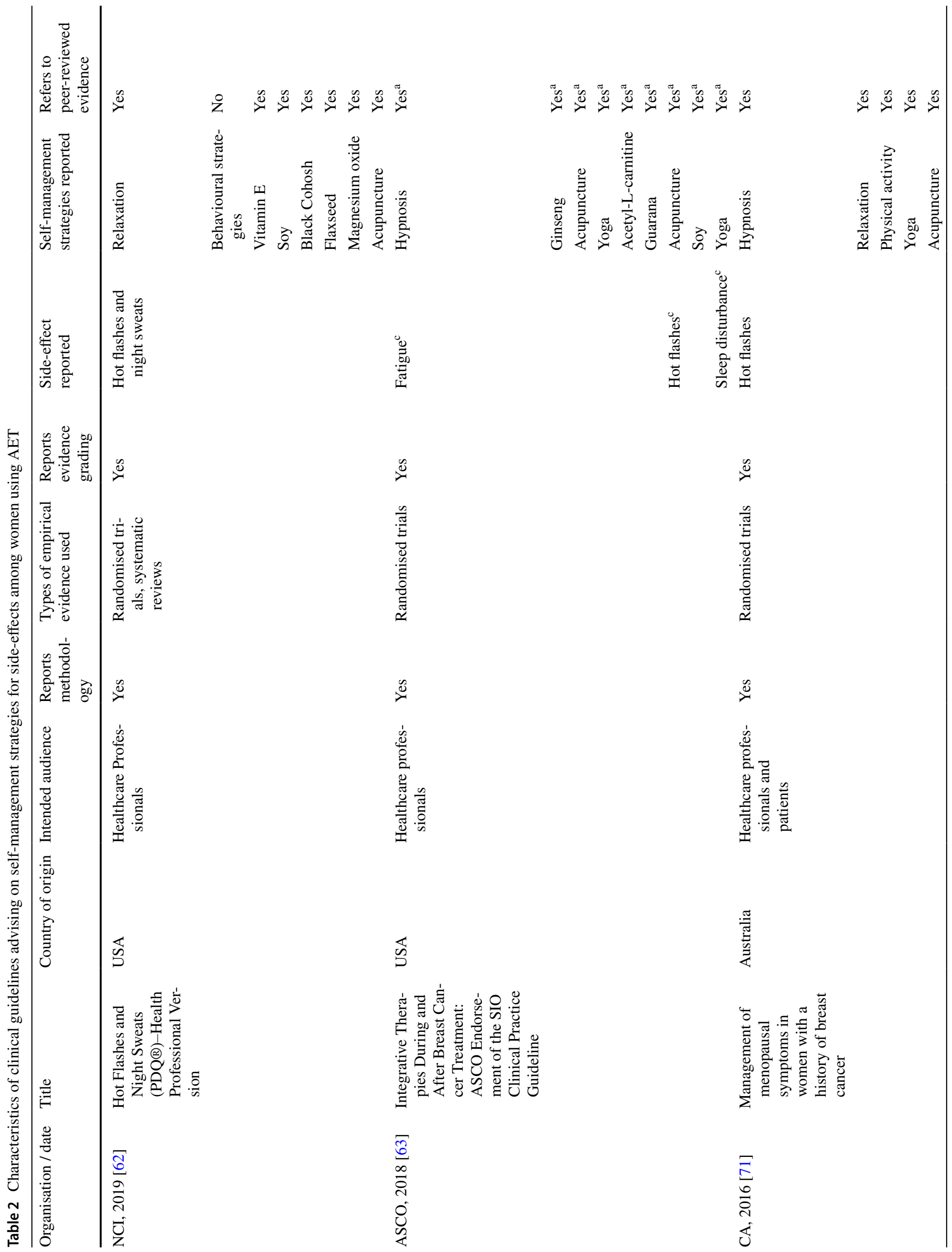




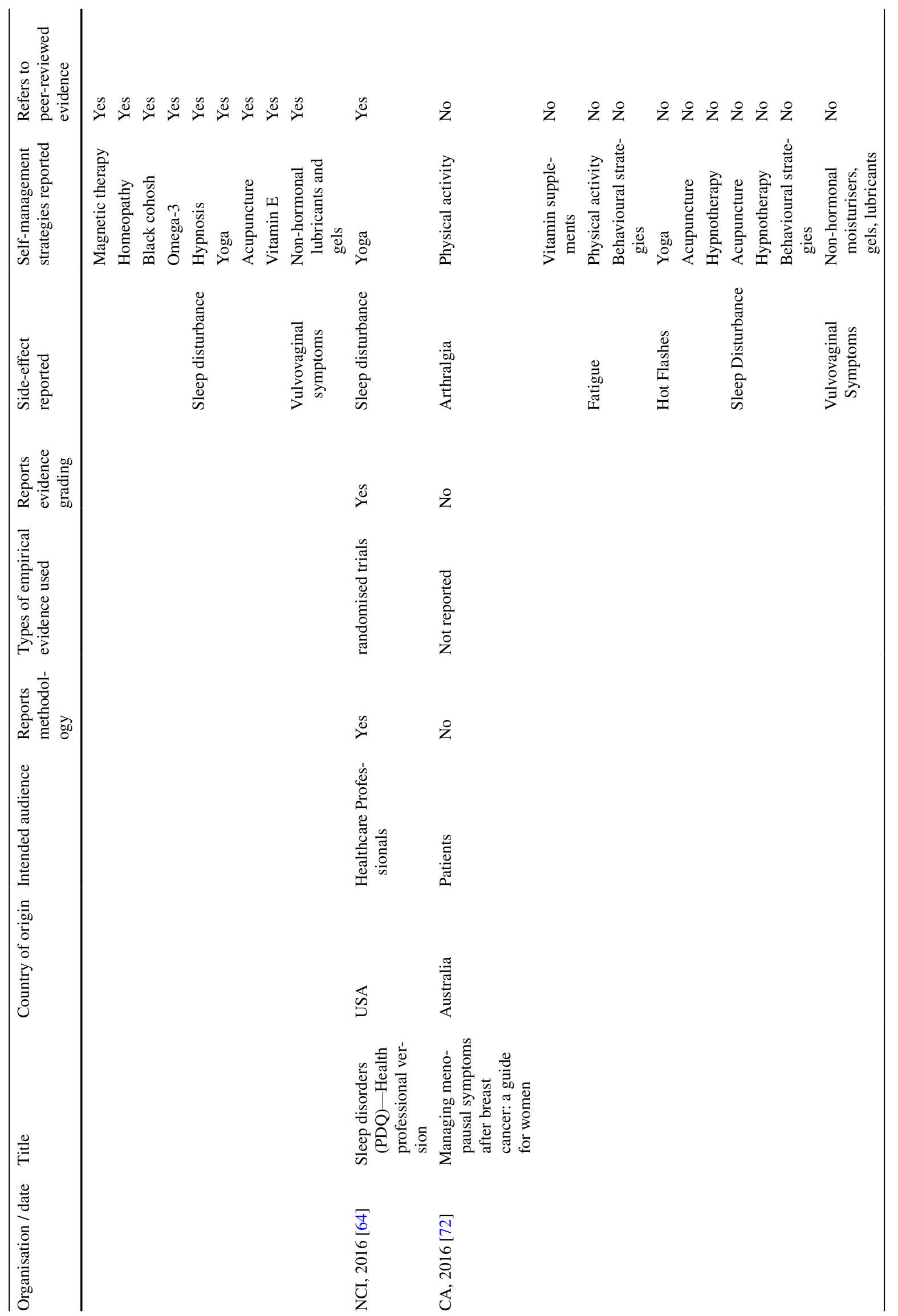




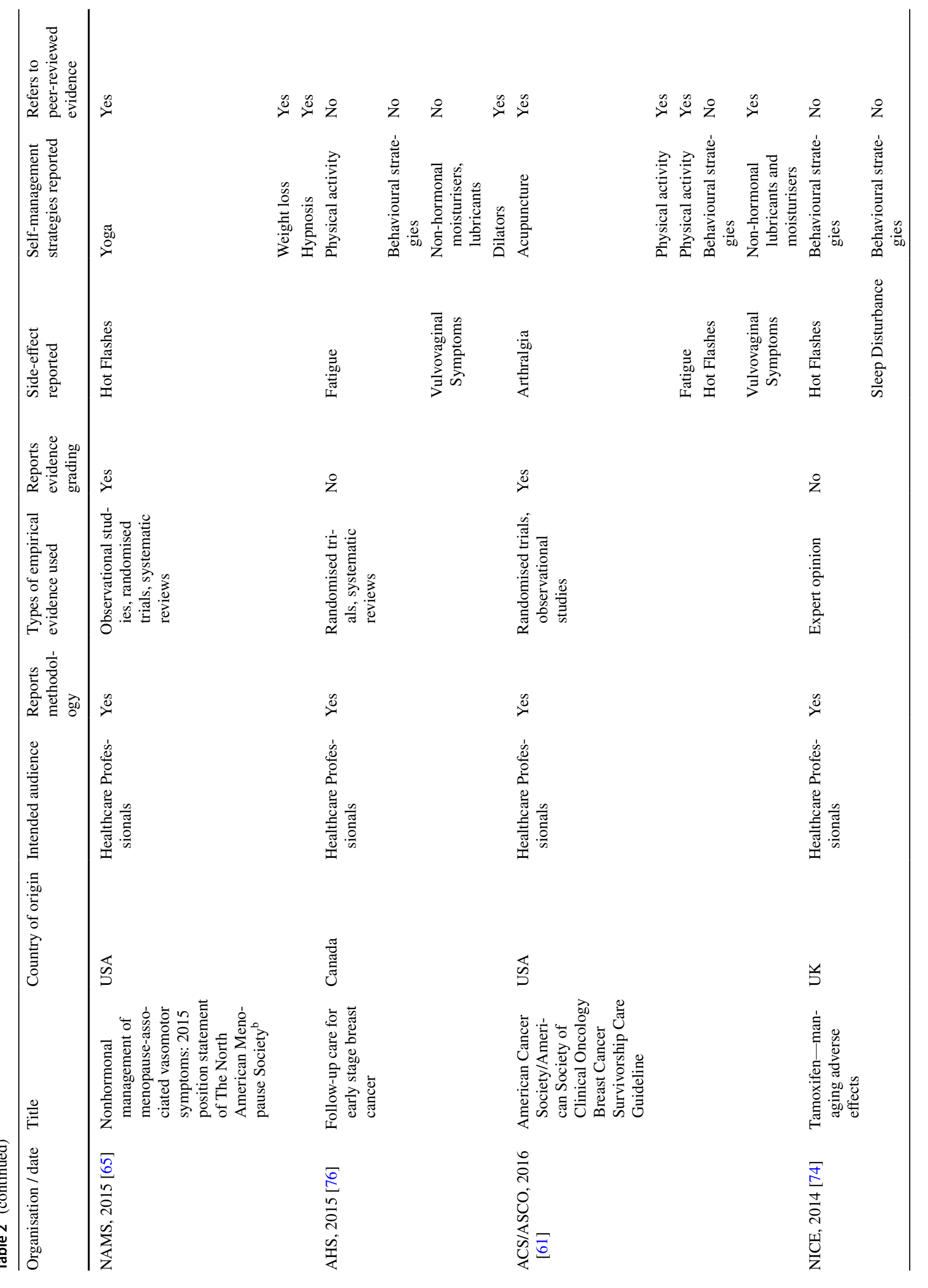




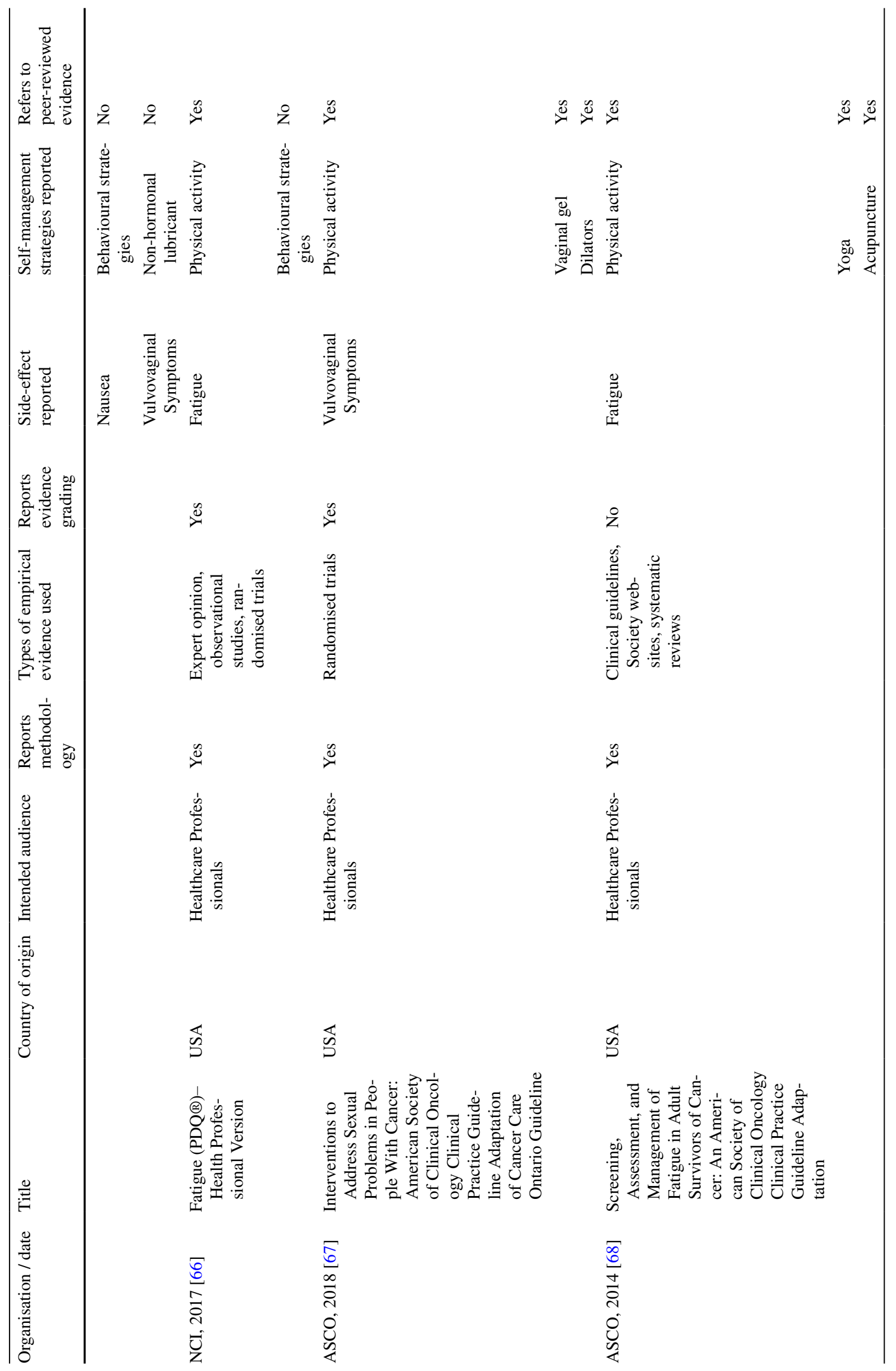




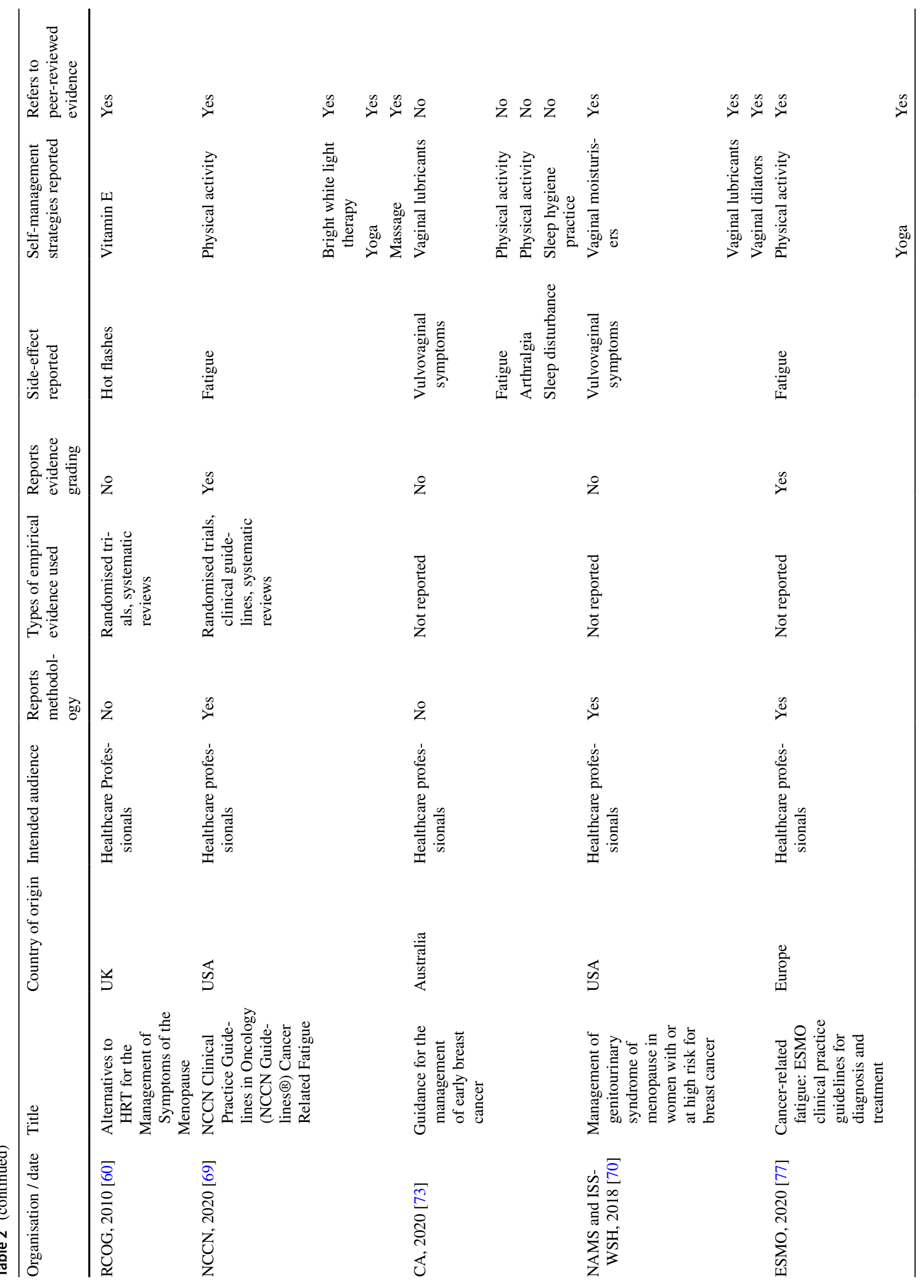




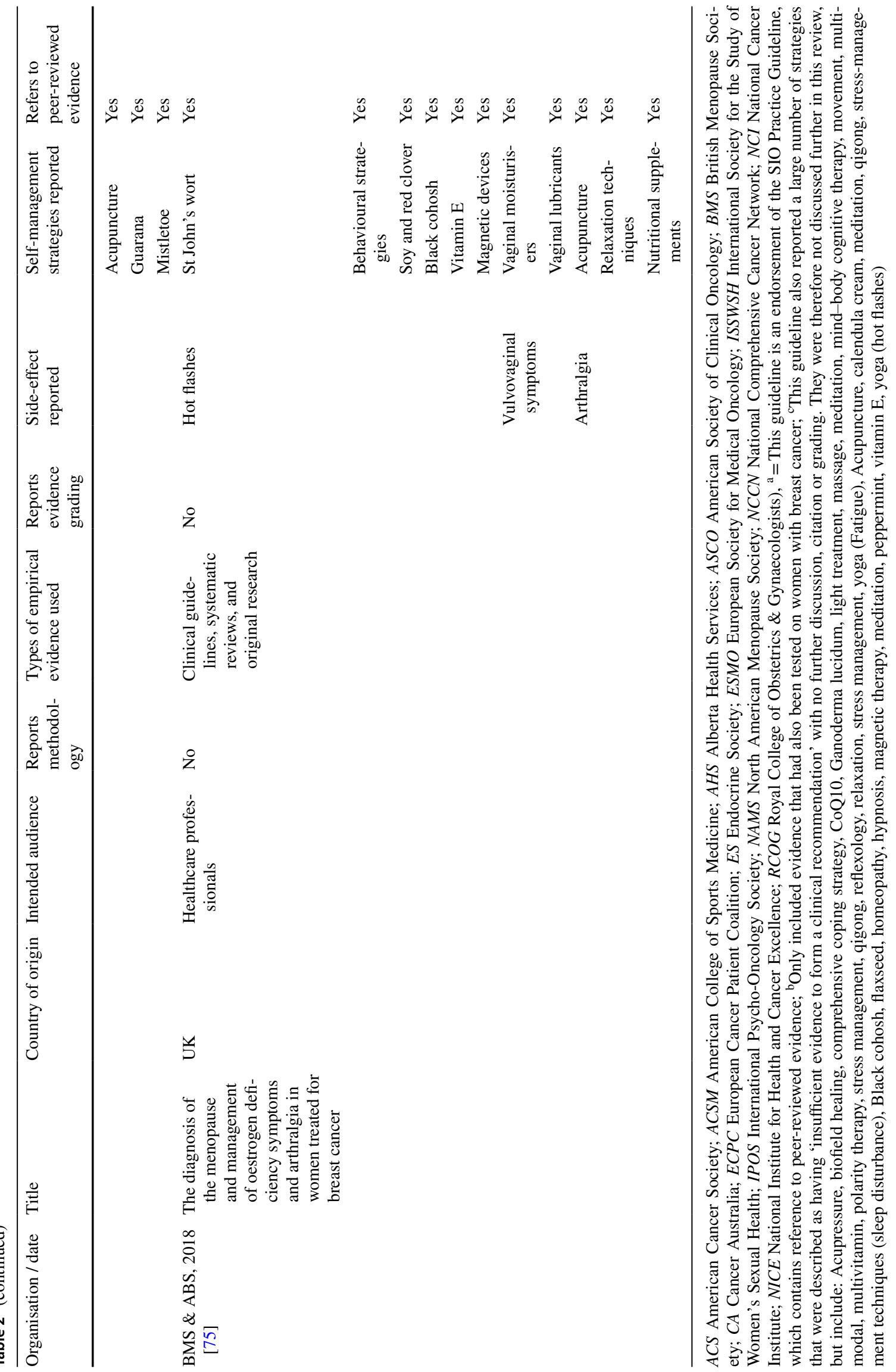




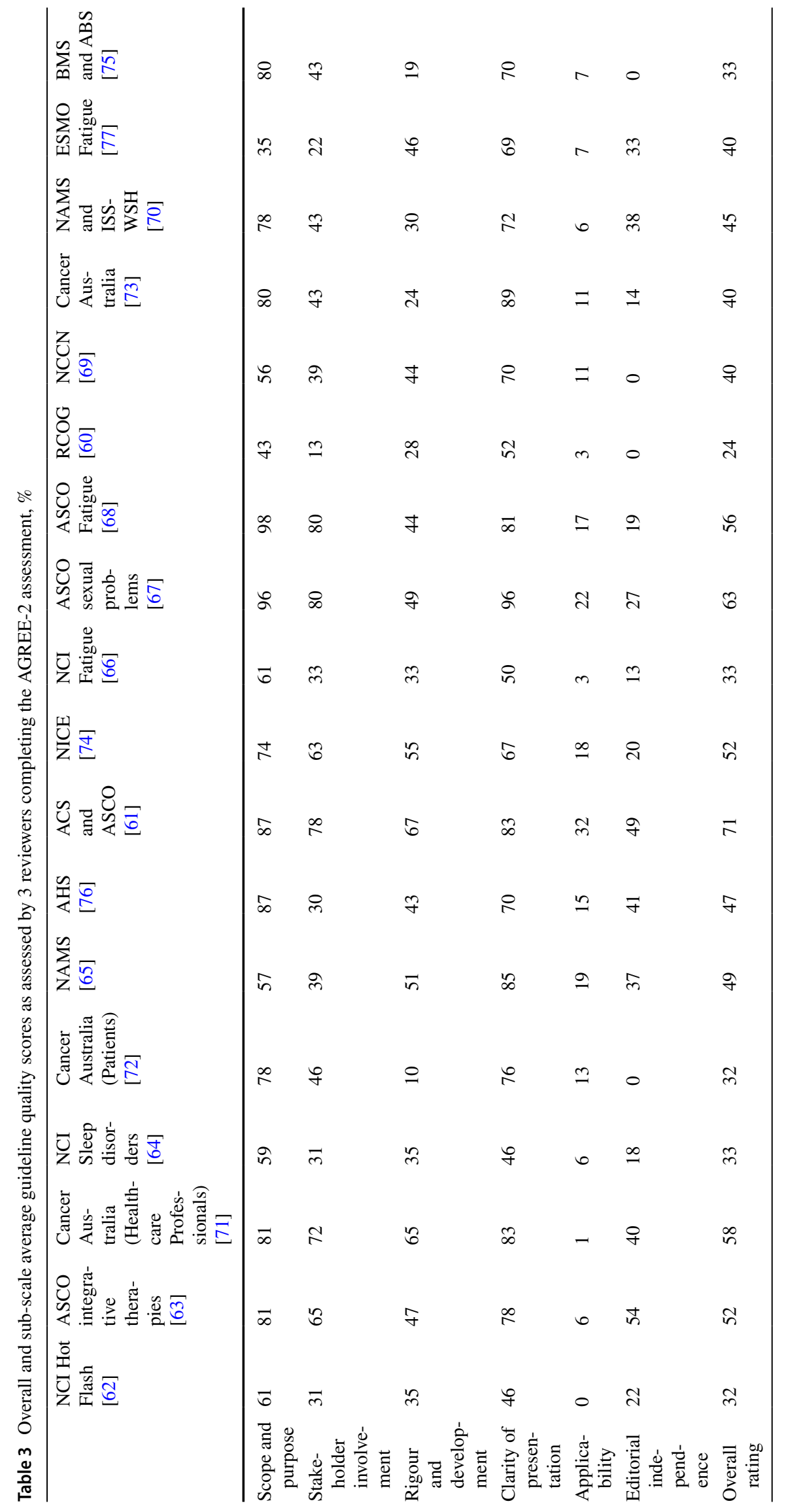


Fifteen systematic reviews used a narrative synthesis [27, 29-31, 35-39, 42, 43, 45, 47, 53, 54], and 18 included at least one meta-analysis [28, 32-34, 40, 41, 44, 46, 48-52, 55-59]. On average, the articles included 7.9 (range of 2-26) relevant primary studies. The most common assessment of methodological quality was the Cochrane Risk of Bias Tool $(n=18)$ [31, 38, 40, 42-45, 47, 49-51, 53-59]. Reporting of adverse events resulting from the strategies evaluated was generally poor, and not reported by 11 systematic reviews [29, 34-37, 39, 47, 48, 52, 55, 58]. Reports from the remaining reviews indicated adverse events were minor (Table 1).

The majority of the guidelines originated in the USA ( $n=10)$ [61-70], with the remainder originating in Australia $(n=3)$ [71-73], the UK $(n=3)[60,74,75]$, Canada $(n=1)$ [76], and Europe $(n=1)$ [77]. Sixteen guidelines were intended for healthcare professionals [60-70, 73-77], one for patients only [72], and one for both healthcare professionals and patients [71]. Fourteen guidelines explicitly reported their methodology for identifying their recommendations [61-71, 74, 76, 77]. When describing self-management strategies, seven referred to systematic reviews $[60,62$, $65,67,68,75,76], 12$ included randomised trials [60-67, $69,71,75,76]$; three referred to observational data $[61,65$, $66]$ and two referred to expert opinion $[66,74]$. One did not report the basis for the recommendation [72]. Ten provided a grading system for the strength of the evidence [61-67, $69,71,77]$.

\section{Arthralgia}

Fourteen systematic reviews [27, 28, 31-33, 39-41, 44, 47-50, 59] and four clinical guidelines [61, 72, 73, 75] reported on five self-management strategies for arthralgia. One review was rated as high quality [59]. The guidelines scored between 32\% [72] and 71\% [61] in terms of quality. An overall summary of findings from the systematic reviews and clinical guidelines is shown in Table 4.4.

\section{Acupuncture}

Eleven systematic reviews reported on the use of acupuncture for arthralgia [27, 28, 31-33, 39-41, 44, 47, 48]. There were no high-quality reviews. Two moderate quality reviews $[40,44]$, one higher quality clinical guideline [61], and one lower quality clinical guideline [75] reported evidence supporting the use of acupuncture for arthralgia, but multiple limitations were noted with studies in this area. These included a tendency to report within group differences rather than between group differences in trial outcomes, short-term follow-up and small sample sizes. A third moderate quality review and meta-analysis reported the effect of acupuncture on arthralgia was not statistically significant in the five trials synthesised.

\section{Physical activity}

Eight systematic reviews [28, 33, 39, 47-50, 59] and three clinical guidelines $[61,72,73]$ reported on physical activity as a self-management strategy for arthralgia. One systematic review [59] and one clinical guideline [61] were rated as high quality. Activities reported included yoga [28, 33, 39, 47, 48, 72], walking [28, 33, 47-49, 59, 72], Nordic walking [33, 48], aerobic and resistance training [28, 33, 39, 47-50, 59, 61, 73], aquatic exercise [33, 49, 72], and Tai-Chi [33]. A high-quality review reported no clear evidence of benefit for exercise therapy to address arthralgia [59]. This finding was supported by most evidence from the lower quality reviews, particularly those focussed on low intensity interventions such as yoga and Tai-Chi. One large randomised controlled trial was included in all systematic reviews [28, 33, 39, 47-50, 59] and one of the clinical guidelines [72], and reported supervised aerobic and resistance training could improve arthralgia in this population.

\section{Supplements}

Two moderate quality systematic reviews [47, 48], two lowquality systematic reviews [33, 39] and a critically lowquality systematic review [28] reported on the use of supplements to address arthralgia. Two lower quality clinical guidelines also reported on the use of supplements for this indication [75], of which one was patient-facing and did not refer to any evidence [72]. Supplements reported included vitamin D [28, 33], vitamin E [33], omega-3 fatty acids [33, $39,47,75]$, Glucosmine sulfate [33, 47, 75], chondroitinsulfate [47], kampo medicine [47], sodium selenite plant enzmes [47], lens culinaris lectin [47], Blue Citrus Herbal [33], high dose vitamin B2 [75], and unspecified vitamins [72]. The weight of evidence suggested supplements are unlikely to be effective at improving arthralgia (Table 4). The majority of primary data referred to within the reviews and guidelines were from weak study designs and small sample sizes. Observational designs tended to yield larger effects in favour of supplement use.

\section{Relaxation}

One lower quality clinical guideline reported evidence from a systematic review and meta-analysis suggesting a moderate to large effect of relaxation techniques on arthralgia [75]. However, no details were provided on techniques or quality of the evidence.

\section{Miscellaneous}

One low-quality systematic review identified a single trial of whole body vibration, which indicated no effect on a range of arthralgia outcomes [39]. 


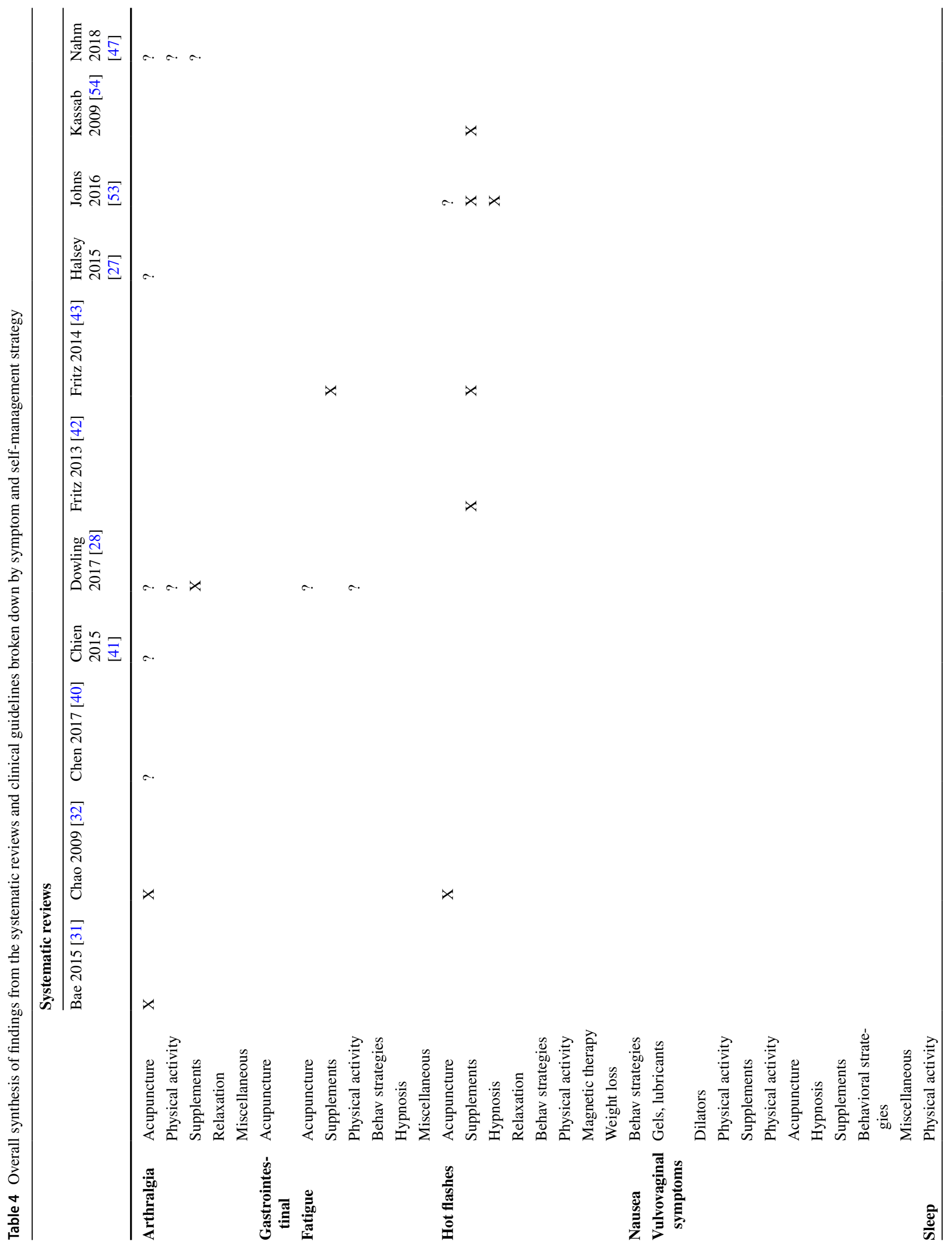




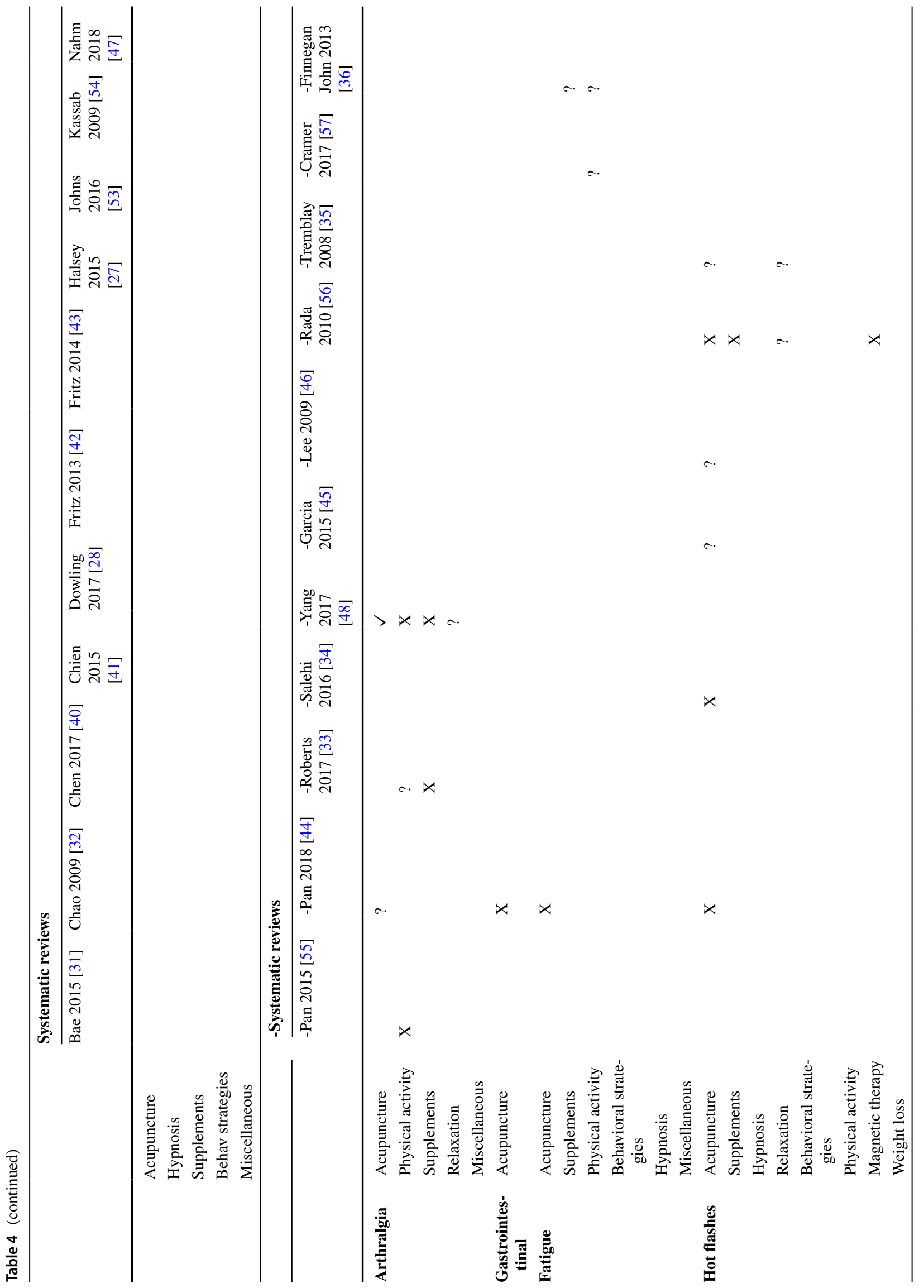




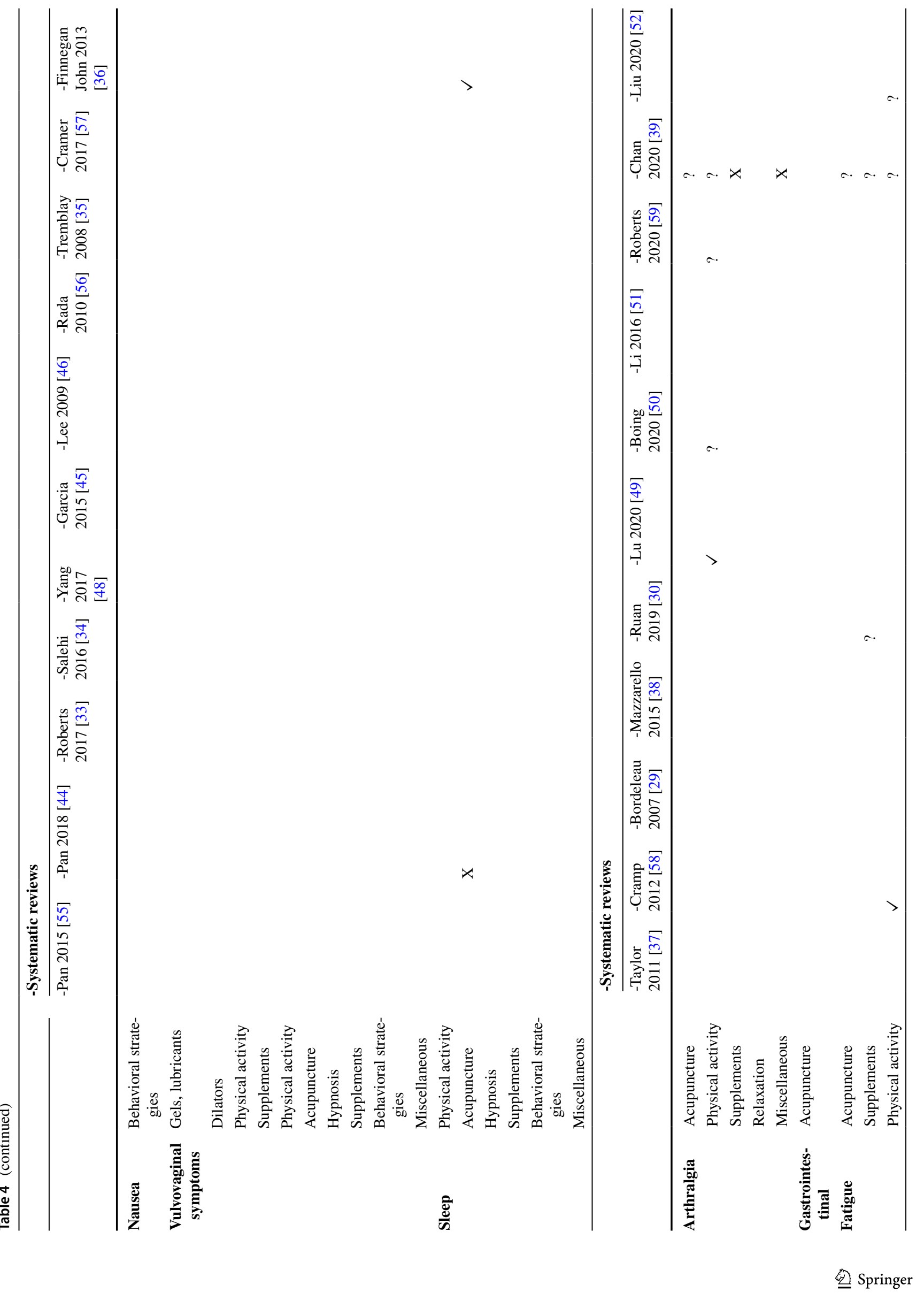




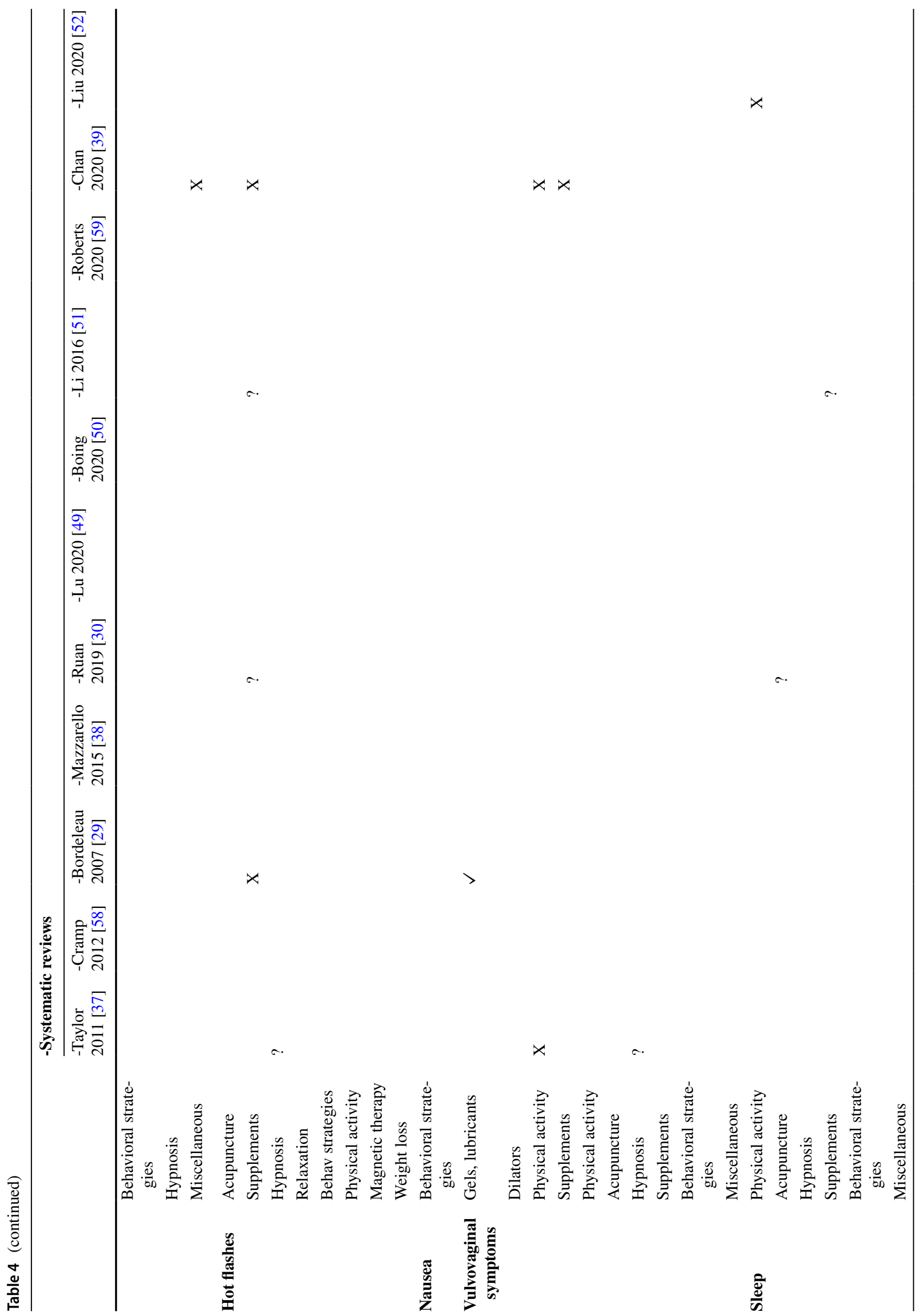




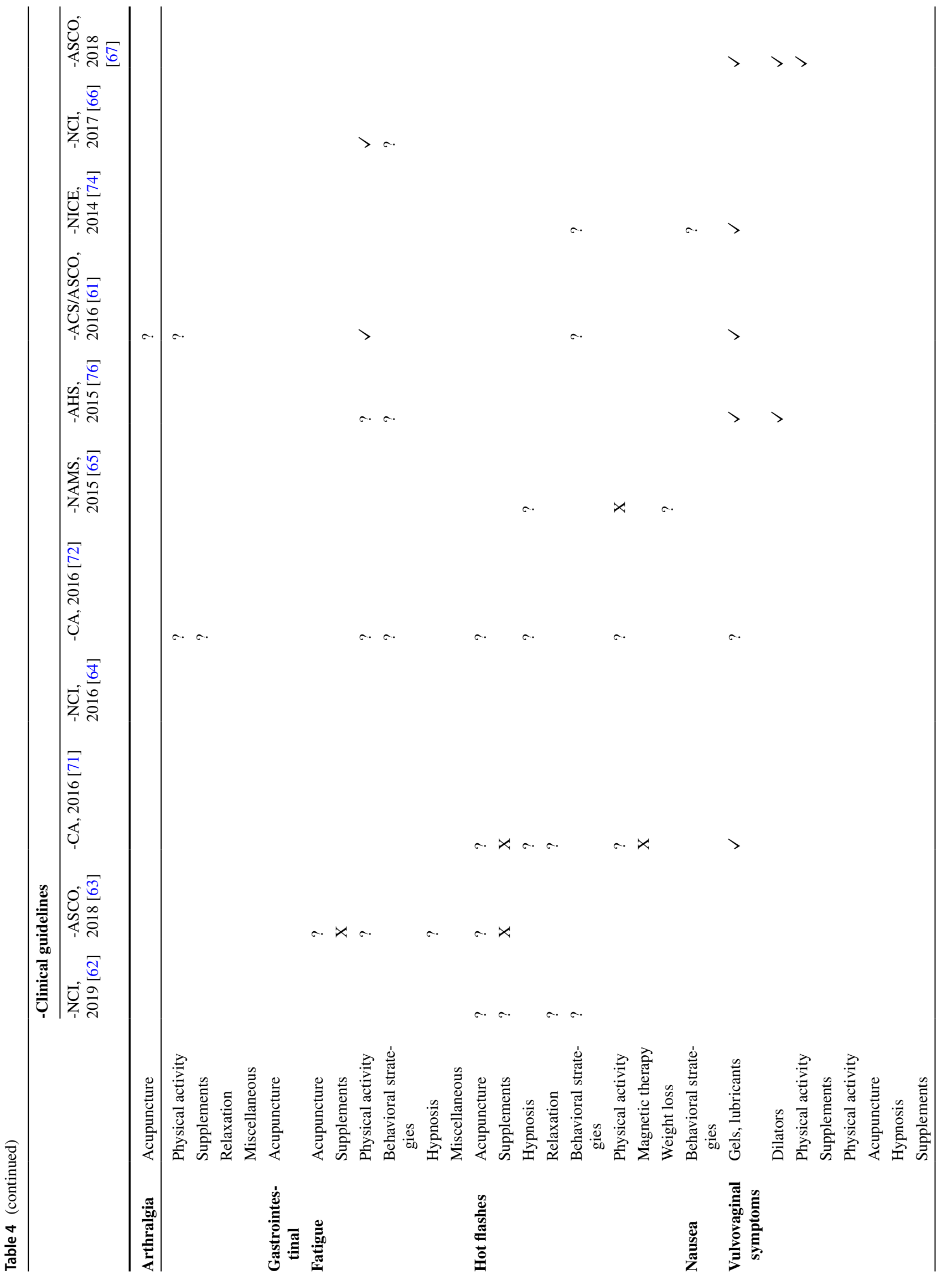




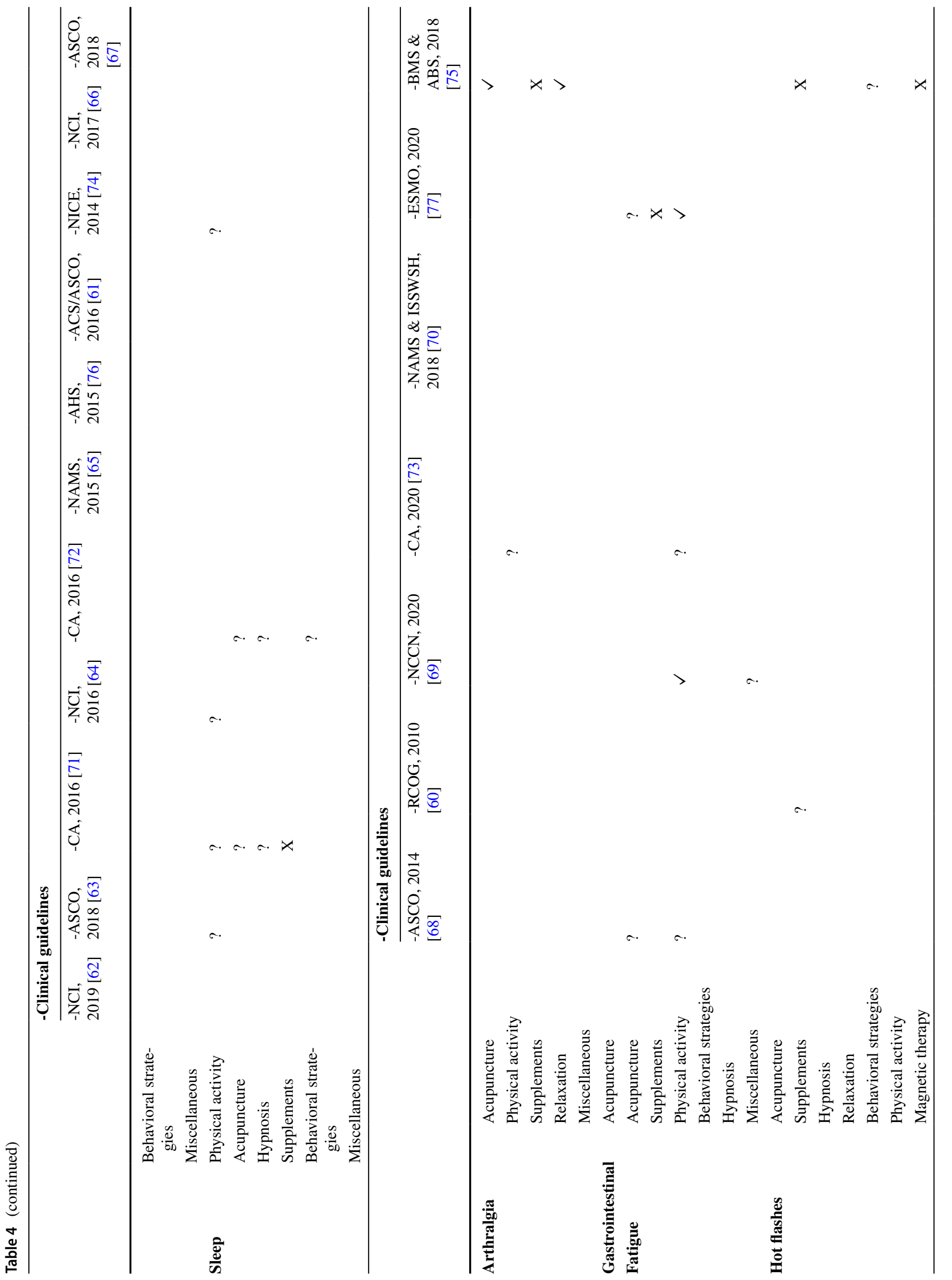




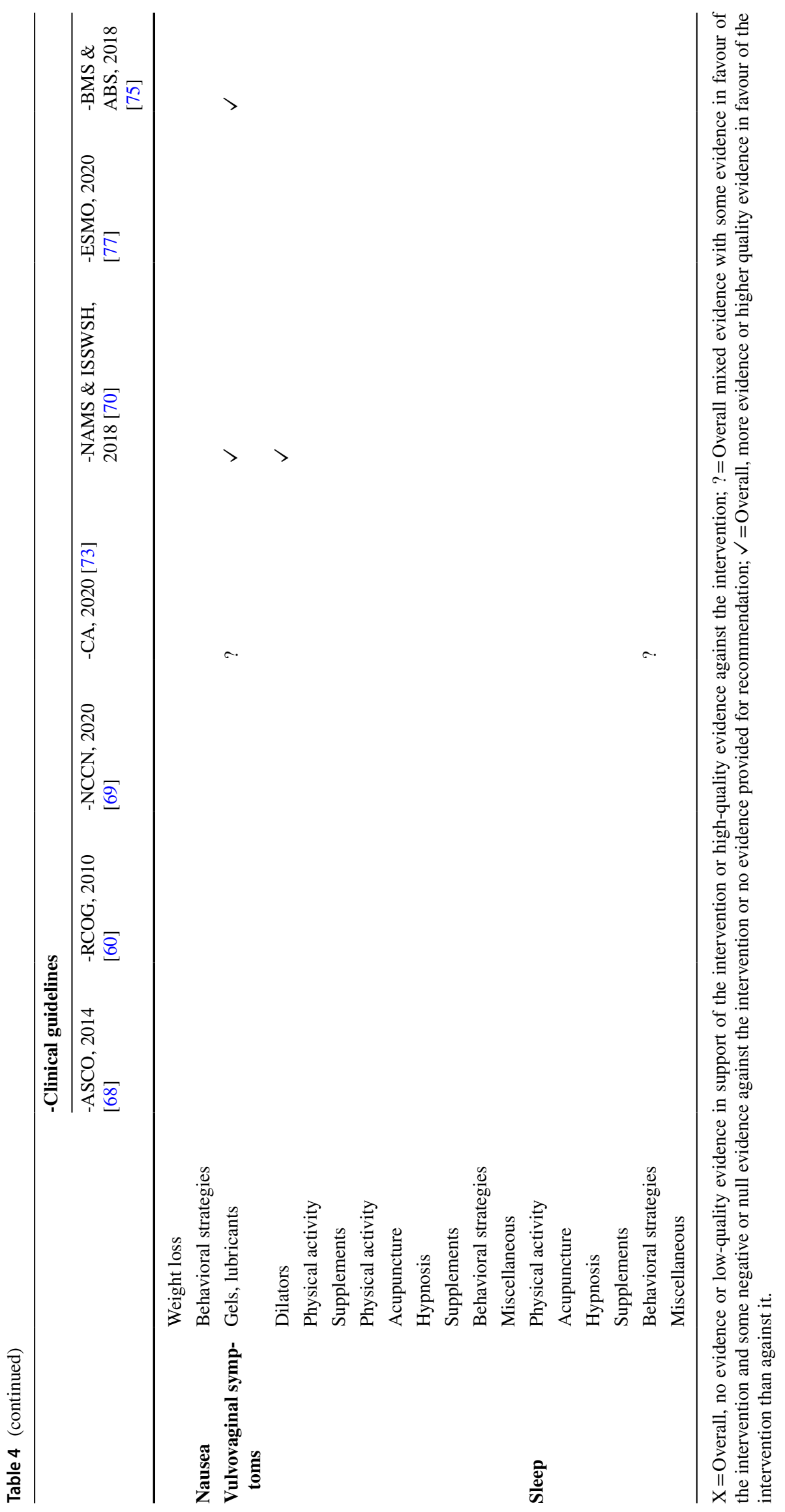




\section{Fatigue}

Eight systematic reviews [28, 36, 39, 43, 44, 55, 57, 58] and nine clinical guidelines $[61,63,66,68,69,72,73,76$, 77] referred to self-management strategies for fatigue. Three systematic reviews were rated as high quality $[55,57,58]$. Three clinical guidelines scored $>50 \%$ with regard to quality $[61,63,68]$.

\section{Acupuncture}

Three systematic reviews [36, 39, 44], two higher quality clinical guidelines $[63,68]$, and one lower quality clinical guideline [77] reported on the use of acupuncture to support fatigue. There were no high-quality reviews. In a moderate quality systematic review and meta-analysis of four studies, acupuncture was reported to be ineffective at managing fatigue in women with breast cancer [44]. One guideline graded acupuncture as ' $\mathrm{C}$ ' whereby 'clinicians are advised to selectively recommend offering acupuncture to patients based on professional judgement and patient preference [63].' A second guideline reported evidence from two randomised trials of acupuncture, indicating it may relieve fatigue, but without an evidence grading [68]. A lower quality guideline reported evidence supporting the use of acupuncture for fatigue, but the guideline panel could not reach a consensus in its recommendation [77].

\section{Supplements}

Three systematic reviews $[30,39,42]$ and two clinical guidelines $[63,77]$ reviewed at least one supplement as a strategy for managing fatigue. A moderate quality systematic review [43] and a critically low-quality systematic review [30] investigated the use of black cohosh to address cancerrelated fatigue. The moderate quality review identified a single observational study, which demonstrated no association between the supplement and fatigue [43]. The low-quality systematic review identified a single randomised controlled trial at high risk of bias, testing Chinese herbs for fatigue [36]. While changes to fatigue were observed in the intervention group, the review concluded this effect should be interpreted with caution. A low-quality systematic review reported data from a single trial indicating spore powder of G. Lucidum improved fatigue in this population [39]. A clinical guideline graded the evidence for Ginseng as 'Grade $\mathrm{C}$ ' indicating equivocal evidence. The same guideline also discouraged the use of Acetyl-L-carntine and Guarana due to moderate or high certainty that there is no net benefit (Grade D) [63]. A lower quality guideline did not recommend guarana or mistletoe extract for fatigue [77].

\section{Physical activity}

Seven systematic reviews $[28,36,39,52,55,57,58]$ and nine clinical guidelines [61, 63, 66, 68, 69, 72, 73, 76, 77] included physical activity interventions for fatigue. One high-quality systematic review investigated the effect of Tai Chi exercise on physical wellbeing, mostly measured using the Chronic Illness Therapy - Fatigue survey [55]. In their meta-analysis of five trials, no overall beneficial effect was observed. This finding is supported by a second high-quality systematic review [58]. A third highquality review concluded yoga could reduce fatigue in the short term but not medium-term compared with no therapy [57]. It also reported there was very low-quality evidence to suggest yoga is comparable to other physical activity interventions. A low-quality review found no evidence for effects of other low intensity physical activity interventions, such as massage therapy [36]. Four clinical guidelines recommended yoga $[63,68,69,77]$ (AGREE-2 range $=40-56 \%$ ).

A high-quality review demonstrated that aerobic exercise was consistently beneficial for fatigue, but there was weak and inconclusive effects for resistance training [58]. One lower quality clinical guideline recommended combining aerobic and resistance exercise (category 1 . High-level evidence and consensus that it was appropriate) [69]. Five guidelines recommended unspecified physical activity $[61,66,68,69,72,73,76]$ (AGREE-2 range $=32-71 \%$ ), although three provided no evidence for this recommendation $[72,73,76]$. In two mixed quality clinical guidelines, the evidence provided was graded as level I (recommendation based on meta-analysis) [61] and level II (recommendation based on non-randomised trials or observational data) [66].

\section{Behavioural strategies}

Three lower quality clinical guidelines recommended a range of strategies, including avoiding long periods in bed [72], consuming enough fruit, vegetables and water [72], and rest $[66,76]$. None were supported by peer-reviewed evidence.

\section{Hypnosis}

One clinical guideline included hypnosis as a strategy for managing fatigue [63] (AGREE-2 score $=52 \%$ ). Its grading (grade $\mathrm{C}$ ) indicated clinicians should selectively offer hypnosis to patients. No systematic reviews were identified for hypnosis and fatigue management. 


\section{Miscellaneous}

A low-quality systematic review reported a single study of neuromuscular taping which demonstrated $\mathrm{s}$ lower fatigue in the intervention group compared with a control group at 5-weeks post-intervention [39]. The same review also reported data from a single trial reporting no effect of whole body vibration on fatigue.

\section{Hot flashes}

Hot flashes were the most common symptom covered by systematic reviews $(n=13)[29,32,34,35,37,42-46,53$, $54,56]$ and clinical guidelines $(n=9)[60-63,65,71,72$, $74,75]$. Three systematic reviews were rated as high quality $[53,54,56]$. Four clinical guidelines were higher quality $[61,63,71,74]$.

\section{Acupuncture}

Eight systematic reviews $[32,34,35,44-46,53,56]$ and four clinical guidelines $[62,63,71,72]$ reported on the use of acupuncture for managing hot flashes. Three systematic reviews were rated as low quality $[32,34,35]$, three as moderate quality [44-46] and two as high quality $[53,56]$. The quality of the guidelines ranged from 32 to $58 \%$. The highquality reviews concluded there was either no evidence for acupuncture for this indication [56] or there was no effect of acupuncture when compared with active pharmacological interventions [53]. Within-group analysis and a lack of longterm follow-up were consistent limitations within trials. The clinical guidelines indicated mixed evidence for acupuncture for managing hot flashes [62, 63, 71, 72] (Table 4).

\section{Supplements}

Nine systematic reviews [29, 30, 39, 42, 43, 51, 53, 54, 56] and five clinical guidelines [60,62, 63, 71, 75] reported 10 different supplements for the self-management of hot flashes. Supplements included vitamin E [29, 53, 56, 60, 62], soy [29, 42, 62, 63, 75], black cohosh [29, 30, 43, 62, 71], Omega 3 [71], Flaxseed [62], magnesium oxide [62], homeopathy [39, 54, 56, 71], herbal medicine [51], medicinal plant extract (guarana) [39], and St John's wort [30, 75]. The overall weight of evidence within the systematic reviews indicated no effect of supplements on hot flashes, with widespread study weaknesses within the field (Table 4).

\section{Hypnosis}

Hypnosis was investigated as a strategy to support hot flashes in two systematic reviews [37, 53], and was also included in three clinical guidelines $[65,71,72]$. A high-quality systematic review identified only one relevant trial that was stopped prematurely due to recruitment difficulties [53]. One higher quality clinical guideline reported the body of evidence was weak and cautiously suggested hypnotherapy could be considered (grade D) [71], and a second lower quality guideline cited the same clinical trial suggesting hypnosis could be a promising strategy for managing hot flashes. They graded the evidence as 'Level 1' (i.e. based on high-quality trials or systematic reviews) [65].

\section{Relaxation}

Two systematic reviews $[35,56]$ and two clinical guidelines $[62,71]$ included relaxation as a strategy for self-managing hot flashes. The systematic reviews were rated as high [56] and low [35] quality. The clinical guidelines scored 32\% [62] and 58\% [71] using the AGREE-2 checklist. Both reviews included two relevant studies, reporting mixed evidence for effects on hot flashes. There was a tendency to report withingroup rather than between-group differences within the individual trials. One guideline reported absent effects in trials with adequate control arms [62], while the other indicated limited evidence of reductions in hot flash frequency and severity [71].

\section{Behavioural strategies}

Four clinical guideline recommended a range of strategies, including lifestyle and environment modifications [61, 62, $74,75]$. Suggestions included avoiding caffeine, alcohol and spicy foods, sipping cool drinks, reducing stress, changing to a cool room temperature, use of portable fans, opening windows, weight loss, wearing loose cotton clothing, and dressing in layers. No evidence was provided for these recommendations.

\section{Physical activity}

A moderate quality systematic review concluded Tai Chi was ineffective for hot flashes [52]. Three clinical guidelines reported on physical activity for hot flashes, all focusing on yoga $[65,71,72]$ (AGREE-2 range $=32-58 \%)$. One guideline indicated no effect of yoga on vasomotor symptoms [65], while a second recommended it without referring to peer-reviewed evidence [72]. The final guideline reported two individual trials among women with breast cancer indicating yoga may reduce the frequency and severity of hot flushes and, when combined with meditation, also improve overall menopausal symptoms [71]. Both trials were assessed by the guideline developers as having a moderate risk of bias. 


\section{Magnetic therapy}

No systematic reviews and two clinical guidelines reported on the use of magnetic therapy for the treatment of hot flashes $[71,75]$ (AGREE-2 scores $=58 \%$ and 33\%). One clinical guideline reported a single randomised controlled trial indicating no effect of magnetic therapy, with the placebo arm reporting a lower frequency of hot flashes at follow-up. This was graded as a ' $C$ ' quality of evidence, indicating cautious application of the recommendation. The second clinical guideline concluded magnetic devices are not advised for this population.

\section{Weight loss}

No systematic reviews were identified reporting on the role of weight loss and hot flashes, and one clinical guideline did report on this [65] (AGREE-2 score $=49 \%$ ). The authors cited a secondary analysis of a randomised dietary trial, indicating women with breast cancer who had lost at least $10 \%$ of their bodyweight during the 2-year follow-up were less likely to report moderate or severe vasomotor symptoms. The guideline indicated the evidence for weight loss on vasomotor symptoms (not distinguishing breast cancer and general population samples) was level II, indicating it may be beneficial for alleviating vasomotor symptoms.

\section{Vulvovaginal symptoms}

Three low-quality systematic reviews were identified for vulvovaginal symptoms [37-39], and self-management strategies to address these symptoms were included in nine clinical guidelines $[61,67,70-76]$. Four guidelines scored $>50 \%$ with regard to quality $[61,67,71,74]$.

\section{Moisturisers, gels, and lubricants}

A low-quality systematic review [38] and nine clinical guidelines of mixed quality $[61,67,70-76]$ all supported the use of moisturisers, gels or lubricants for this indication. Three guidelines did not refer to peer-reviewed literature [72-74]. The systematic review reported reductions in vaginal dryness and dyspareunia when using a $\mathrm{pH}$-balanced gel and a vaginal moisturiser [38], and reduced dyspareunia when using aqueous lidocaine. A higher quality clinical guideline recommended the use of lubricants for all sexual touch in addition to frequent application of vaginal moisturiser [67]. A second higher quality clinical guideline reported a $1 \mathrm{~A}$ level of evidence (based on RCTs) for non-hormonal waterbased lubricants and moisturisers for vaginal dryness [61].

\section{Vaginal dilators}

Three clinical guidelines recommended the use of dilators to address vaginismus and vaginal stenosis $[67,70,76]$. It was not clear whether these recommendations were informed by primary research undertaken with women affected by breast cancer.

\section{Physical activity}

A systematic review rated as low quality [37] and a higher quality clinical guideline [67] reported on the evidence for physical activity and vulvovaginal symptoms. The systematic review reported two randomised trials of exercise interventions that had no effect on sexual functioning. The clinical guideline focussed on general exercise and pelvic floor exercises and reported these may be effective to support vulvovaginal discomfort and lower urinary tract symptoms. It was unclear what primary research informed this recommendation.

\section{Supplements}

A low-quality systematic review reported there was no effect of medicinal plant extract (guarana) on sexual function [39].

\section{Sleep disturbance}

Six systematic reviews of varying quality $[30,39,44,51$, $52,57]$ and five clinical guidelines [63, 71-74] reported on strategies to address sleep disturbance in women with breast cancer. Three guidelines scored $>50 \%$ with regard to quality $[63,71,74]$.

\section{Physical activity}

A high-quality systematic review identified eight relevant studies comparing yoga to no therapy $(n=6)$ or psychological interventions $(n=2)$ [57]. It concluded yoga is favourable in comparison with no therapy, but not psychological interventions for sleep disturbance. A moderate-quality systematic review also indicated there was no long-term effect of Tai Chi on sleep [52]. A low-quality systematic review reported physical activity interventions may improve several sleep-related outcomes, although the interventions were poorly defined [39]. A clinical guideline reported there was equivocal evidence or small net benefit (grade C) for gentle yoga in improving sleep quality [63] (AGREE-2 score $=52 \%)$. 


\section{Acupuncture}

A moderate quality systematic review reported a single acupuncture trial demonstrating this intervention did not significantly improve sleep quality [44]. Two clinical guidelines included acupuncture for sleep disturbance [71, 72], although only one referred to peer-reviewed evidence [71]. This guideline cited a single randomised trial, which indicated acupuncture improved sleep quality compared with sham acupuncture, but only within group differences were reported.

\section{Hypnosis}

Two clinical guidelines included hypnosis for sleep disturbance, although only one referred to peer-reviewed evidence [71, 72]. This reported a single randomised trial with a moderate risk of bias indicating hypnotherapy improved sleep quality when compared with a no treatment control [71].

\section{Supplements}

A low-quality systematic review reported herbal medicine may alleviate insomnia [51]. A systematic review rated as critically low quality reported black cohosh (with or without St John's wort) may be effective at improving sleep disturbance. A higher quality clinical guideline reported a single randomised trial comparing gabapentin with vitamin E over 12 weeks [71]. Their findings indicated vitamin $E$ had no effect on sleep disturbance compared with gabapentin.

\section{Behavioural strategies}

Three clinical guidelines recommended a range of behavioural strategies to improve sleep quality, although none were supported by peer-reviewed evidence [72-74]. These included sleep hygiene techniques, such as not exercising late in the day, maintaining a regular bedtime, avoiding stimulants (e.g. caffeine, alcohol), restricting the bedroom for sleep, avoiding napping, and keeping the bedroom cool.

\section{Miscellaneous}

A low-quality systematic review reported neuromuscular taping did not reduce sleep disturbance [39].

\section{Gastrointestinal discomfort}

One high-quality systematic review and meta-analysis reported on the use of acupuncture to manage gastrointestinal discomfort [44]. No clinical guidelines were identified.

\section{Acupuncture}

Five studies were included in the meta-analysis, which showed no benefit of acupuncture on gastrointestinal discomfort [44].

\section{Nausea}

One clinical guideline reported on the topic of nausea induced by AET [74] (AGREE-2 score =52\%). No systematic reviews were identified.

\section{Behavioural strategies}

The NICE clinical guideline recommended that women should take tamoxifen with food or milk, or at night [74]. No empirical evidence was provided.

\section{Discussion}

In this umbrella review of clinical guidelines and systematic reviews, there was no clear evidence to support the majority of self-management strategies currently recommended and used by breast cancer survivors experiencing side-effects from AET. Unanimous agreement was only observed for moisturisers, lubricants and gels for women affected by vulvovaginal symptoms, where a systematic review and seven clinical guidelines endorsed this strategy. Considering the prevalence of side-effects of AET [2-6], and that over a quarter of women with these symptoms attempt selfmanagement, there should be a renewed focus in evaluating and implementing support for breast cancer survivors [6]. Healthcare professionals supporting the management of breast cancer survivors should counsel women that the evidence supporting most self-management strategies for AET side-effects have a weak and inconsistent evidence base. While many strategies are low risk, patients should be encouraged to seek healthcare professional support if they do not alleviate their symptoms, as evidence supporting pharmacological interventions appears stronger [78].

Despite the wide-ranging benefits of physical activity observed among cancer populations [79-81], and its inclusion in multiple clinical guidelines identified here $[61,63$, $65,66,68,69,71-73,76,77]$, there was often weak or inconclusive evidence from systematic reviews to support 
this strategy for arthralgia, fatigue, hot flashes, vulvovaginal symptoms and sleep disturbance in women affected by breast cancer using AET. This was particularly the case for low intensity activities such as Tai Chi and yoga. Among the high-quality systematic reviews, yoga [57] and aerobic exercise [58] was shown to be effective for fatigue, although yoga may only be beneficial in the short-term. The conclusion that the evidence for physical activity for self-managing AET symptoms is weak or inconclusive was made on the basis that the majority of the primary research informing these reviews was affected by either low statistical power, poor study design or inadequate follow-up. A rare example is a trial led by Irwin and colleagues [82] which strongly influenced the evidence to support physical activity for arthralgia within the reviews and guidelines. Such trials suggest it would be premature to conclude that physical activity is ineffective for self-managing AET, but similar high-quality studies are needed. Specifically, further information is needed to inform precise recommendations for physical activity type, duration and intensity of physical activity. Reporting guidelines such as the Consensus on Exercise Reporting Template (CERT) could be also more widely used to improve the specificity of reporting [83].

We aimed to observe the extent to which guidelines adequately reflected scientific evidence generated in systematic reviews. Strategies with at least some supporting evidence for a beneficial effect were generally appropriately reflected in the clinical guidelines, as indicated by moisturisers, gels and lubricants, and physical activity. However, there were examples of clinical guidelines introducing recommended strategies not supported by systematic review evidence. For example, weight loss and physical activity for hot flashes, hypnosis for fatigue and sleep, and behavioural strategies for fatigue, hot flashes, and sleep. In the case of dilators for vulvovaginal symptoms, the clinical guidelines largely relied on narrative reviews and early phase pilot trials, and our search did not identify any systematic reviews in this area. A systematic review of dilators for this indication could therefore be warranted.

The reviews and guidelines identified observed consistent weaknesses with the primary research they included, which often led us to conclude that the evidence for strategies was inconclusive. The use of acupuncture for arthralgia is a useful example to illustrate this point. Weak study designs were common, including observational studies with a high risk of bias and underpowered pilot trials that relied upon withingroup rather than between-group comparisons. Short-term follow-up within clinical trials was also widespread, which can limit the extent to which these data apply to women using AET over a 5- to 10-year period [84]. These weaknesses were common, but not ubiquitous, and the mixed evidence may explain why others have concluded this strategy to be efficacious for this indication [78]. Adequately powered trials of promising supportive care interventions with long-term follow-up are needed to inform clinical guidelines.

There were also weaknesses with regard to the reviews that we identified. Overall, 13 (39\%) systematic reviews were rated as either low or critically low quality. Our finding that duplicate data extraction was only present in less than half of the reviews is particularly problematic. There was a high degree of overlap with regard to the strategies assessed for specific indications within the systematic reviews. For example, we identified 11 systematic reviews reporting on the use of acupuncture for arthralgia. This is a form of research waste, as evidenced by so few clinical guidelines including acupuncture for arthralgia management. This duplication could be addressed through pre-registration of systematic review protocols.

The quality of some clinical guidelines was also low, and they often made recommendations without citing evidence or reporting evidence for strategies that had only been tested in healthy populations or different clinical groups. Most guidelines failed to consider potential barriers to implementation within routine clinical care. Guideline developers should consider describing the resource implications of applying recommendations and suggest audit criteria to monitor and drive implementation. Furthermore, editorial independence was assessed as being particularly poor for the guidelines identified. To improve transparency within guideline development, the views of the funding body and competing interests of the contributors should be disclosed and easy to identify.

Our decision to include both systematic reviews and clinical guidelines enabled us to produce a more comprehensive review with a broader range of self-management strategies. However, a limitation that should be acknowledged is that synthesizing the data from each document type simultaneously was often challenging because the purpose of each document is inherently different. For example, the clinical guidelines endorsing physical activity were likely to consider the wide-ranging benefits observed among cancer populations [79-81], and therefore their recommendations may not have been based solely on the evidence for selfmanaging symptoms of AET. This wider context should be considered when comparing guidelines with the findings of systematic reviews. Our ability to compare each document type was further limited by the differences between the quality assessment tool we used for each, making the quality scores difficult to compare. We included all reviews and guidelines regardless of the quality scores they were given. While we gave greater weight to the higher quality reviews and guidelines within our narrative synthesis, different conclusions may have been drawn had we excluded publications considered to be lower quality.

Further limitations are that we excluded pharmacological approaches and strategies that would require healthcare 
professional supervision, many of which have known efficacy [78]. Patients should be encouraged to seek healthcare professional support if the initial self-management strategies are ineffective. For pragmatic reasons we did not search all databases for the clinical guidelines, and this may also have led us to miss important documents. Side-effects such as nausea and fatigue are associated with a range of breast cancer treatments, but our review focussed on literature involving women using AET. Effective self-management interventions tested among women experiencing these symptoms who are not using AET could also be useful, but may require evaluation before being widely recommended. Finally, a limitation of the umbrella review method overall is that too much weight is placed on primary studies that are cited across multiple reviews and guidelines. This should be considered when interpreting our findings.

In conclusion, we identified a large body of systematic reviews and clinical guidelines of self-management strategies for women experiencing side-effects from AET. However, unanimous agreement for a strategy was only observed for moisturisers, gels and lubricants for women experiencing vulvovaginal symptoms. Evidence supporting physical activity for AET symptoms was only clear for aerobic activity and yoga for alleviating fatigue. Breast cancer survivors should therefore be cautious about using most self-management strategies currently recommended for AET side-effects. Healthcare professionals should be aware that although the risk of harm is unlikely with most of these strategies, the likelihood of benefit is often unclear. Primary research in this area relied heavily on inadequately powered trials using poor study designs. A large proportion of the systematic reviews and clinical guidelines identified were also of lower quality. There is a need for high-quality longitudinal primary research of promising self-management interventions for women with breast cancer using AET. Findings from such trials should be incorporated within clinical guidelines that have considered how to implement these strategies into routine practice.

Supplementary Information The online version contains supplementary material available at https://doi.org/10.1007/s11764-021-01114-7.

Acknowledgements We would like to acknowledge the support of Erin Raine in screening the clinical guidelines. We also acknowledge the ACTION investigators: Rachael Ellison, Michelle Collinson, Ellen Mason Amanda Farrin, Robbie Foy, Suzanne Hartley, Ellen Mason, Jamie Metherell, Richard Neal, Duncan Petty, Julie Saritag, Galina Velikova, Michael Wolf.

Author Contribution Conceptualization $=\mathrm{SS}, \mathrm{LH}, \mathrm{NK}, \mathrm{CG}, \mathrm{JC}$; data curation $=\mathrm{NK}, \mathrm{SS}, \mathrm{LH}, \mathrm{RF}, \mathrm{RN}, \mathrm{CG}, \mathrm{EB}, \mathrm{CG}, \mathrm{KL}$; formal analysis: $\mathrm{SS}, \mathrm{LH}, \mathrm{KL}, \mathrm{AB}$; funding acquisition $=\mathrm{SS}, \mathrm{CG}, \mathrm{RN}, \mathrm{JC}$; investigation methodology $=\mathrm{SS}, \mathrm{NK}, \mathrm{LH}$; project administration $=\mathrm{SS}, \mathrm{LH}, \mathrm{NK}$; Resources $=$ SS; Software $=$ SS, NK; Supervision $=$ SS, NK, LH; original draft $=\mathrm{SS}, \mathrm{LH}, \mathrm{AB}, \mathrm{CG}$; review and editing $=$ all authors; review of submitted manuscript: all.
Funding The study was funded by Yorkshire Cancer Research (funder ref: L417) (PI: Smith and Graham). Smith also acknowledges the support of a Yorkshire Cancer Research Fellowship. This report is independent research supported by the National Institute for Health Research NIHR Advanced Fellowship, Dr Samuel Smith NIHR300588. The views expressed in this publication are those of the author(s) and not necessarily those of the NHS, the National Institute for Health Research or the Department of Health and Social Care. The funders had no role in the design of the study, data collection, analysis, interpretation of data, and in the writing of this manuscript.

Data availability Template data collection forms, data extracted from included studies, and any other materials used in the review are available from the corresponding author upon reasonable request.

Code availability Not applicable.

\section{Declarations}

Conflict of interest The authors declare no competing interests.

Ethical approval Not applicable.

Consent to participate Not applicable.

Consent for publication Not applicable.

Open Access This article is licensed under a Creative Commons Attribution 4.0 International License, which permits use, sharing, adaptation, distribution and reproduction in any medium or format, as long as you give appropriate credit to the original author(s) and the source, provide a link to the Creative Commons licence, and indicate if changes were made. The images or other third party material in this article are included in the article's Creative Commons licence, unless indicated otherwise in a credit line to the material. If material is not included in the article's Creative Commons licence and your intended use is not permitted by statutory regulation or exceeds the permitted use, you will need to obtain permission directly from the copyright holder. To view a copy of this licence, visit http://creativecommons.org/licenses/by/4.0/.

\section{References}

1. Bray F, Ferlay J, Soerjomataram I, Siegel RL, Torre LA, Jemal A. Global cancer statistics 2018: GLOBOCAN estimates of incidence and mortality worldwide for 36 cancers in 185 countries. CA Cancer J Clin. 2018;68:394-424. https://doi.org/10.3322/caac. 21492.

2. Cella D, Fallowfield LJ. Recognition and management of treatment-related side effects for breast cancer patients receiving adjuvant endocrine therapy. Breast Cancer Res Treat. 2008;107:16780. https://doi.org/10.1007/s10549-007-9548-1.

3. Howell A, Cuzick J, Baum M, Buzdar A, Dowsett M, Forbes JF, et al. Results of the ATAC (Arimidex, Tamoxifen, Alone or in Combination) trial after completion of 5 years' adjuvant treatment for breast cancer. Lancet. 2005;365:60-2. https://doi.org/10.1016/ S0140-6736(04)17666-6.

4. Jakesz R, Jonat W, Gnant M, Mittlboeck M, Greil R, Tausch $\mathrm{C}$, et al. Switching of postmenopausal women with endocrineresponsive early breast cancer to anastrozole after 2 years' adjuvant tamoxifen: combined results of ABCSG trial 8 and ARNO 
95 trial. Lancet. 2005;366:455-62. https://doi.org/10.1016/S01406736(05)67059-6.

5. Breast International Group (BIG) 1-98 Collaborative Group, Thürlimann B, Keshaviah A, Coates AS, Mouridsen H, Mauriac $\mathrm{L}$, et al. A comparison of letrozole and tamoxifen in postmenopausal women with early breast cancer. N Engl J Med 2005; 353 : 2747-57. https://doi.org/10.1056/NEJMoa052258.

6. Peate M, Saunders C, Cohen P, Hickey M. Who is managing menopausal symptoms, sexual problems, mood and sleep disturbance after breast cancer and is it working? Findings from a large community-based survey of breast cancer survivors. Breast Cancer Res Treat. 2021. https://doi.org/10.1007/s10549-021-06117-7.

7. Fallowfield L, Cella D, Cuzick J, Francis S, Locker G, Howell A. Quality of life of postmenopausal women in the Arimidex, Tamoxifen, Alone or in Combination (ATAC) adjuvant breast cancer trial. J Clin Oncol. 2004;22:4261-71. https://doi.org/10. 1200/JCO.2004.08.029.

8. Ganz PA, Petersen L, Bower JE, Crespi CM. Impact of adjuvant endocrine therapy on quality of life and symptoms: observational data over 12 Months from the mind-body study. J Clin Oncol. 2016;34:816-24. https://doi.org/10.1200/JCO.2015.64.3866.

9. Schover LR, Baum GP, Fuson LA, Brewster A, Melhem-Bertrandt A. Sexual problems during the first 2 years of adjuvant treatment with aromatase inhibitors. J Sex Med. 2014;11:3102-11. https:// doi.org/10.1111/jsm.12684.

10. Demissie S, Silliman RA, Lash TL. Adjuvant tamoxifen: predictors of use, side effects, and discontinuation in older women. J Clin Oncol. 2001;19:322-8. https://doi.org/10.1200/JCO.2001. 19.2.322.

11. Kahn KL, Schneider EC, Malin JL, Adams JL, Epstein AM. Patient centered experiences in breast cancer: predicting longterm adherence to tamoxifen use. Med Care. 2007;45:431-9. https://doi.org/10.1097/01.mlr.0000257193.10760.7f.

12. Murphy CC, Bartholomew LK, Carpentier MY, Bluethmann SM, Vernon SW. Adherence to adjuvant hormonal therapy among breast cancer survivors in clinical practice: a systematic review. Breast Cancer Res Treat. 2012;134:459-78. https://doi.org/10. 1007/s10549-012-2114-5.

13. Brett J, Boulton M, Fenlon D, Hulbert-Williams NJ, Walter FM, Donnelly P, et al. Adjuvant endocrine therapy after breast cancer: a qualitative study of factors associated with adherence. Patient Prefer Adherence. 2018;12:291-300. https://doi.org/10.2147/PPA. S145784.

14. Wheeler SB, Roberts MC, Bloom D, Reeder-Hayes KE, Espada M, Peppercorn J, et al. Oncology providers' perspectives on endocrine therapy prescribing and management. Patient Prefer Adherence. 2016;10:2007-19. https://doi.org/10.2147/PPA.S95594.

15. Winn AN, Dusetzina SB. The association between trajectories of endocrine therapy adherence and mortality among women with breast cancer. Pharmacoepidemiol Drug Saf. 2016;25:953-9. https://doi.org/10.1002/pds.4012.

16. McCowan C, Wang S, Thompson AM, Makubate B, Petrie DJ. The value of high adherence to tamoxifen in women with breast cancer: a community-based cohort study. Br J Cancer. 2013;109:1172-80. https://doi.org/10.1038/bjc.2013.464.

17. Makubate B, Donnan PT, Dewar JA, Thompson AM, McCowan C. Cohort study of adherence to adjuvant endocrine therapy, breast cancer recurrence and mortality. Br J Cancer. 2013;108:1515-24. https://doi.org/10.1038/bjc.2013.116.

18. Hershman DL, Kushi LH, Shao T, Buono D, Kershenbaum A, Tsai WY, et al. Early discontinuation and nonadherence to adjuvant hormonal therapy in a cohort of 8,769 early-stage breast cancer patients. J Clin Oncol. 2010;28:4120-8. https://doi.org/10.1200/ JCO.2009.25.9655.

19. Yood MU, Owusu C, Buist DSM, Geiger AM, Field TS, Thwin SS, et al. Mortality impact of less-than-standard therapy in older breast cancer patients. J Am Coll Surg. 2008;206:66-75. https:// doi.org/10.1016/j.jamcollsurg.2007.07.015.

20. Early Breast Cancer Trialists' Collaborative Group (EBCTCG), Davies C, Godwin J, Gray R, Clarke M, Cutter D, et al. Relevance of breast cancer hormone receptors and other factors to the efficacy of adjuvant tamoxifen: patient-level meta-analysis of randomised trials. Lancet 2011;378:771-84. https://doi.org/10. 1016/S0140-6736(11)60993-8.

21. Haque R, Ahmed SA, Fisher A, Avila CC, Shi J, Guo A, et al. Effectiveness of aromatase inhibitors and tamoxifen in reducing subsequent breast cancer. Cancer Med. 2012;1:318-27. https:// doi.org/10.1002/cam4.37.

22. Aromataris E, Fernandez R, Godfrey C, Holly C, Khalil H, Tungpunkom P. Chapter 10: Umbrella Reviews. JBI Man. Evid. Synth., 2020.

23. Page MJ, McKenzie JE, Bossuyt PM, Boutron I, Hoffmann TC, Mulrow CD, et al. The PRISMA 2020 statement: an updated guideline for reporting systematic reviews. BMJ. 2021;372: n71. https://doi.org/10.1136/bmj.n71.

24. McGowan J, Sampson M, Salzwedel DM, Cogo E, Foerster V, Lefebvre C. PRESS Peer Review of Electronic Search Strategies: 2015 Guideline Statement. J Clin Epidemiol. 2016;75:40-6. https://doi.org/10.1016/j.jclinepi.2016.01.021.

25. Shea BJ, Reeves BC, Wells G, Thuku M, Hamel C, Moran J, et al. AMSTAR 2: a critical appraisal tool for systematic reviews that include randomised or non-randomised studies of healthcare interventions, or both. BMJ 2017; 358. https://doi.org/10.1136/ bmj.j4008.

26. Brouwers MC, Kho ME, Browman GP, Burgers JS, Cluzeau F, Feder G, et al. AGREE II: advancing guideline development, reporting and evaluation in health care. CMAJ Can Med Assoc J. 2010;182:E839-42. https://doi.org/10.1503/cmaj.090449.

27. Halsey EJ, Xing M, Stockley RC. Acupuncture for joint symptoms related to aromatase inhibitor therapy in postmenopausal women with early-stage breast cancer: a narrative review. Acupunct Med. 2015;33:188-95. https://doi.org/10.1136/acupmed-2014-010735.

28. Dowling M, McDonagh B, Meade E. Arthralgia in breast cancer survivors: an integrative review of endocrine therapy. Oncol Nurs Forum 2017; 44. https://doi.org/10.1188/17.337-349.

29. Bordeleau L, Pritchard K, Goodwin P, Loprinzi C. Therapeutic options for the management of hot flashes in breast cancer survivors: an evidence-based review. Clin Ther. 2007;29:230-41. https://doi.org/10.1016/j.clinthera.2007.02.006.

30. Ruan X, Mueck AO, Beer A-M, Naser B, Pickartz S. Benefitrisk profile of black cohosh (isopropanolic Cimicifuga racemosa extract) with and without St John's wort in breast cancer patients. Climacteric. 2019;22:339-47. https://doi.org/10.1080/13697137. 2018.1551346.

31. Bae K, Yoo H-S, Lamoury G, Boyle F, Rosenthal DS, Oh B. Acupuncture for aromatase inhibitor-induced arthralgia. Integr Cancer Ther. 2015;14:496-502.

32. Chao L-F, Zhang AL, Liu H-E, Cheng M-H, Lam H-B, Lo SK. The efficacy of acupoint stimulation for the management of therapy-related adverse events in patients with breast cancer: a systematic review. Breast Cancer Res Treat. 2009;118:255-67. https://doi.org/10.1007/s10549-009-0533-8.

33. Roberts K, Rickett K, Greer R, Woodward N. Management of aromatase inhibitor induced musculoskeletal symptoms in postmenopausal early Breast cancer: A systematic review and metaanalysis. Crit Rev Oncol Hematol. 2017;111:66-80. https://doi. org/10.1016/j.critrevonc.2017.01.010.

34. Salehi A, Marzban M, Zadeh AR. Acupuncture for treating hot flashes in breast cancer patients: an updated meta-analysis. Support Care Cancer. 2016;24:4895-9. https://doi.org/10.1007/ s00520-016-3345-5. 
35. Tremblay A, Sheeran L, Aranda SK. Psychoeducational interventions to alleviate hot flashes: a systematic review. Menopause. 2008;15:192-202. https://doi.org/10.1097/gme.0b013e3180 $5 \mathrm{c} 08 \mathrm{dc}$.

36. Finnegan-John J, Molassiotis A, Richardson A, Ream E. A systematic review of complementary and alternative medicine interventions for the management of cancer-related fatigue. Integr Cancer Ther. 2013;12:276-90. https://doi.org/10.1177/1534735413 485816.

37. Taylor S, Harley C, Ziegler L, Brown J, Velikova G. Interventions for sexual problems following treatment for breast cancer: a systematic review. Breast Cancer Res Treat. 2011;130:711-24. https://doi.org/10.1007/s10549-011-1722-9.

38. Mazzarello S, Hutton B, Ibrahim MFK, Jacobs C, Shorr R, Smith $S$, et al. Management of urogenital atrophy in breast cancer patients: a systematic review of available evidence from randomized trials. Breast Cancer Res Treat. 2015;152:1-8. https://doi. org/10.1007/s10549-015-3434-z.

39. Chan CWH, Tai D, Kwong S, Chow KM, Chan DNS, Law BMH. The effects of pharmacological and non-pharmacological interventions on symptom management and quality of life among breast cancer survivors undergoing adjuvant endocrine therapy: a systematic review. Int J Environ Res Public Health 2020;17. https://doi.org/10.3390/ijerph17082950.

40. Chen L, Lin C-C, Huang T-W, Kuan Y-C, Huang Y-H, Chen $\mathrm{H}-\mathrm{C}$, et al. Effect of acupuncture on aromatase inhibitor-induced arthralgia in patients with breast cancer: A meta-analysis of randomized controlled trials. Breast. 2017;33:132-8. https://doi.org/ 10.1016/j.breast.2017.03.015.

41. Chien T-J, Liu C-Y, Chang Y-F, Fang C-J, Hsu C-H. Acupuncture for treating aromatase inhibitor-related arthralgia in breast cancer: a systematic review and meta-analysis. J Altern Complement Med. 2015;21:251-60. https://doi.org/10.1089/acm.2014.0083.

42. Fritz H, Seely D, Flower G, Skidmore B, Fernandes R, Vadeboncoeur S, et al. Soy, Red Clover, and Isoflavones and Breast Cancer: A Systematic Review. PLoS ONE. 2013;8: e81968. https://doi. org/10.1371/journal.pone.0081968.

43. Fritz H, Seely D, McGowan J, Skidmore B, Fernandes R, Kennedy DA, et al. Black Cohosh and Breast Cancer: A Systematic Review. Integr Cancer Ther. 2014;13:12-29. https://doi.org/10.1177/15347 35413477191.

44. Pan Y, Yang K, Shi X, Liang H, Shen X, Wang R, et al. Clinical Benefits of Acupuncture for the Reduction of Hormone TherapyRelated Side Effects in Breast Cancer Patients: A Systematic Review. Integr Cancer Ther. 2018;17:602-18. https://doi.org/10. $1177 / 1534735418786801$.

45. Garcia MK, Graham-Getty L, Haddad R, Li Y, McQuade J, Lee RT, et al. Systematic review of acupuncture to control hot flashes in cancer patients: Acupuncture for Hot Flashes. Cancer. 2015;121:3948-58. https://doi.org/10.1002/cncr.29630.

46. Lee MS, Kim K-H, Choi S-M, Ernst E. Acupuncture for treating hot flashes in breast cancer patients: a systematic review. Breast Cancer Res Treat. 2009;115:497-503. https://doi.org/10.1007/ s10549-008-0230-z.

47. Nahm N, Mee S, Marx G. Efficacy of management strategies for aromatase inhibitor-induced arthralgia in breast cancer patients: a systematic review. Asia Pac J Clin Oncol. 2018;14:374-82. https://doi.org/10.1111/ajco.12845.

48. Yang GS, Kim HJ, Griffith KA, Zhu S, Dorsey SG, Renn CL. Interventions for the Treatment of Aromatase Inhibitor-Associated Arthralgia in Breast Cancer Survivors: A Systematic Review and Meta-analysis. Cancer Nurs. 2017;40:E26-41. https://doi.org/10. 1097/NCC.0000000000000409.

49. Lu G, Zheng J, Zhang L. The effect of exercise on aromatase inhibitor-induced musculoskeletal symptoms in breast cancer survivors: a systematic review and meta-analysis. Support Care Cancer. 2020;28:1587-96. https://doi.org/10.1007/ s00520-019-05186-1.

50. Boing L, Vieira M de CS, Moratelli J, Bergmann A, Guimarães AC de A. Effects of exercise on physical outcomes of breast cancer survivors receiving hormone therapy - A systematic review and meta-analysis. Maturitas 2020; 141: 71-81. https://doi.org/ 10.1016/j.maturitas.2020.06.022.

51. Li Y, Zhu X, Bensussan A, Li P, Moylan E, Delaney G, et al. Herbal Medicine for Hot Flushes Induced by Endocrine Therapy in Women with Breast Cancer: A Systematic Review and Meta-Analysis. Evid-Based Complement Altern Med. 2016;2016:1327251. https://doi.org/10.1155/2016/1327251.

52. Liu L, Tan H, Yu S, Yin H, Baxter GD. The effectiveness of tai chi in breast cancer patients: A systematic review and meta-analysis. Complement Ther Clin Pract. 2020;38: 101078. https://doi.org/ 10.1016/j.ctcp.2019.101078.

53. Johns C, Seav SM, Dominick SA, Gorman JR, Li H, Natarajan $\mathrm{L}$, et al. Informing hot flash treatment decisions for breast cancer survivors: a systematic review of randomized trials comparing active interventions. Breast Cancer Res Treat. 2016;156:415-26. https://doi.org/10.1007/s10549-016-3765-4.

54. Kassab S, Cummings M, Berkovitz S, van Haselen R, Fisher P. Homeopathic medicines for adverse effects of cancer treatments. Cochrane Database Syst Rev. 2009. https://doi.org/10.1002/14651 858.CD004845.pub2.

55. Pan Y, Yang K, Shi X, Liang H, Zhang F, Lv Q. Tai Chi Chuan Exercise for Patients with Breast Cancer: A Systematic Review and Meta-Analysis. Evid Based Complement Alternat Med. 2015;2015:1-15. https://doi.org/10.1155/2015/535237.

56. Rada G, Capurro D, Pantoja T, Corbalán J, Moreno G, Letelier LM, et al. Non-hormonal interventions for hot flushes in women with a history of breast cancer. Cochrane Database Syst Rev. 2010. https://doi.org/10.1002/14651858.CD004923.pub2.

57. Cramer H, Lauche R, Klose P, Lange S, Langhorst J, Dobos GJ. Yoga for improving health-related quality of life, mental health and cancer-related symptoms in women diagnosed with breast cancer. Cochrane Database Syst Rev. 2017. https://doi.org/10. 1002/14651858.CD010802.pub2.

58. Cramp F, Byron-Daniel J. Exercise for the management of cancer-related fatigue in adults. Cochrane Database Syst Rev. 2012. https://doi.org/10.1002/14651858.CD006145.pub3.

59. Roberts KE, Rickett K, Feng S, Vagenas D, Woodward NE. Exercise therapies for preventing or treating aromatase inhibitor-induced musculoskeletal symptoms in early breast cancer. Cochrane Database Syst Rev. 2020. https://doi.org/10.1002/14651 858.CD012988.pub2.

60. Royal College of Obstetricians and Gynaecologists. Alternatives to HRT for the management of symptoms of the menopause. 2010.

61. Runowicz CD, Leach CR, Henry NL, Henry KS, Mackey HT, Cowens-Alvarado RL, et al. American Cancer Society/American Society of Clinical Oncology Breast Cancer Survivorship Care Guideline. J Clin Oncol. 2016;34:611-35. https://doi.org/10.1200/ JCO.2015.64.3809.

62. National Cancer Institute. Hot flashes and night sweats (PDQ(R)) - Health professional version. Bethesda, MD: National Cancer Institute; 2019. Available from: https://www.cancer.gov/aboutcancer/treatment/side-effects/hot-flashes-hp-pdq\#_56

63. Lyman GH, Greenlee H, Bohlke K, Bao T, DeMichele AM, Deng GE, et al. Integrative Therapies During and After Breast Cancer Treatment: ASCO Endorsement of the SIO Clinical Practice Guideline. J Clin Oncol. 2018;36:2647-55. https://doi.org/10. 1200/JCO.2018.79.2721.

64. National Cancer Institute. Sleep disorders (PDQ(R)) - Health professional version. Bethesda, MD: National Cancer Institute; 2016. 
Available from: https://www.cancer.gov/about-cancer/treatment/ side-effects/sleep-disorders-hp-pdq

65. Nonhormonal management of menopause-associated vasomotor symptoms. 2015 position statement of The North American Menopause Society. Menopause. 2015;22:1155-74. https://doi. org/10.1097/GME.0000000000000546.

66. National Cancer Institute. Fatigue (PDQ(R)) - Health professional version. Bethesda, MD: National Cancer Institute; 2017. Available from: https://www.cancer.gov/about-cancer/treatment/side-effects/ fatigue/fatigue-hp-pdq

67. Carter J, Lacchetti C, Andersen BL, Barton DL, Bolte S, Damast $\mathrm{S}$, et al. Interventions to Address Sexual Problems in People With Cancer: American Society of Clinical Oncology Clinical Practice Guideline Adaptation of Cancer Care Ontario Guideline. J Clin Oncol. 2018;36:492-511. https://doi.org/10.1200/JCO.2017.75. 8995.

68. Bower JE, Bak K, Berger A, Breitbart W, Escalante CP, Ganz PA, et al. Screening, Assessment, and Management of Fatigue in Adult Survivors of Cancer: An American Society of Clinical Oncology Clinical Practice Guideline Adaptation. J Clin Oncol. 2014;32:1840-50. https://doi.org/10.1200/JCO.2013.53.4495.

69. National Comprehensive Cancer Network. Cancer-Related Fatigue. 2020. Available from: https://www.nccn.org/profession als/physician_gls/pdf/fatigue.pdf

70. Faubion SS, Larkin LC, Stuenkel CA, Bachmann GA, Chism LA, Kagan R, et al. Management of genitourinary syndrome of menopause in women with or at high risk for breast cancer: consensus recommendations from The North American Menopause Society and The International Society for the Study of Women's Sexual Health. Menopause N Y N. 2018;25:596-608. https:// doi.org/10.1097/GME.0000000000001121.

71. Cancer Australia. Management of menopausal symptoms in women with a history of breast cancer. Surry Hills, New South Wales, Australia: Cancer Australia; 2016. Available from: https://www.canceraustralia.gov.au/publications-and-resources/ clinical-practice-guidelines/menopausal-guidelines

72. Cancer Australia. Managing menopausal symptoms after breast cancer: a guide for women. Surry Hills, New South Wales, Australia: Cancer Australia; 2016. Available from: https:// www.canceraustralia.gov.au/publications-and-resources/ cancer-australia-publications/managing-menopausal-sympt oms-after-breast-cancer-guide-women

73. Cancer Australia. Guidance for the management of early breast cancer. Recommendations and practice points. Surry Hills, New South Wales, Australia: 2020. Available from: https://www. canceraustralia.gov.au/publications-and-resources/cancer-austr alia-publications/guidance-management-early-breast-cancerrecommendations-and-practice-points

74. National Institute for Health and Care Excellence. Clinical knowledge summary: Tamoxifen - managing adverse effects.
London, UK: 2014. Available from: https://cks.nice.org.uk/topics/tamoxifen-managing-adverse-effects/

75. British Menopause Society, Association of Breast Surgeons. The diagnosis of the menopause and management of oestrogen deficiency symptoms and arthralgia in women treated for breast cancer. 2018. Available from: https://associationofbr eastsurgery.org.uk/media/64908/11-bms-consensusstatementthe-diagnosis-of-the-menopause-and-management-of-oestr ogen-deficiency-symptoms-and-arthralgia-in-women-treatedfor-breast-cancer-for-abs-01c.pdf

76. Alberta Health Services. Follow-up care for early stage breast cancer. 2015. Available from: https://www.albertahealthse rvices.ca/assets/info/hp/cancer/if-hp-cancer-guide-br013-earlystage-follow-up.pdf

77. Fabi A, Bhargava R, Fatigoni S, Guglielmo M, Horneber M, Roila F, et al. Cancer-related fatigue: ESMO Clinical Practice Guidelines for diagnosis and treatment. Ann Oncol. 2020;31:713-23. https://doi.org/10.1016/j.annonc.2020.02.016.

78. Franzoi MA, Agostinetto E, Perachino M, Mastro LD, Azambuja E de, Vaz-Luis I, et al. Evidence-based approaches for the management of side-effects of adjuvant endocrine therapy in patients with breast cancer. Lancet Oncol 2021;0. https://doi. org/10.1016/S1470-2045(20)30666-5.

79. Brown JC, Winters-Stone K, Lee A, Schmitz KH. Cancer, Physical Activity, and Exercise. Compr Physiol. 2012;2:2775-809. https://doi.org/10.1002/cphy.c120005.

80. de Rezende LFM, de Sá TH, Markozannes G, Rey-López JP, Lee I-M, Tsilidis KK, et al. Physical activity and cancer: an umbrella review of the literature including 22 major anatomical sites and 770000 cancer cases. Br J Sports Med. 2018;52:82633. https://doi.org/10.1136/bjsports-2017-098391.

81. Rock CL, Thomson C, Gansler T, Gapstur SM, McCullough ML, Patel AV, et al. American Cancer Society guideline for diet and physical activity for cancer prevention. CA Cancer J Clin. 2020;70:245-71. https://doi.org/10.3322/caac.21591.

82. Irwin ML, Cartmel B, Gross CP, Ercolano E, Li F, Yao X, et al. Randomized exercise trial of aromatase inhibitor-induced arthralgia in breast cancer survivors. J Clin Oncol. 2015;33:1104-11. https://doi.org/10.1200/JCO.2014.57.1547.

83. Slade SC, Dionne CE, Underwood M, Buchbinder R. Consensus on Exercise Reporting Template (CERT): Explanation and Elaboration Statement. Br J Sports Med. 2016;50:1428-37. https://doi. org/10.1136/bjsports-2016-096651.

84. Burstein HJ, Lacchetti C, Anderson H, Buchholz TA, Davidson NE, Gelmon KA, et al. Adjuvant Endocrine Therapy for Women With Hormone Receptor-Positive Breast Cancer: ASCO Clinical Practice Guideline Focused Update. J Clin Oncol. 2018;37:42338. https://doi.org/10.1200/JCO.18.01160.

Publisher's Note Springer Nature remains neutral with regard to jurisdictional claims in published maps and institutional affiliations. 